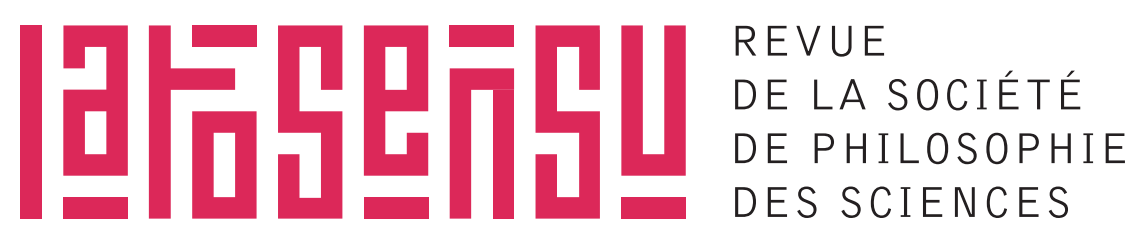

Vol $4 N^{\circ} 12017$

DOI http://dx.doi.org/10.20416/lsrsps.v4i1.513

\title{
Olivier Ouzilou
}

\section{Alínnation}
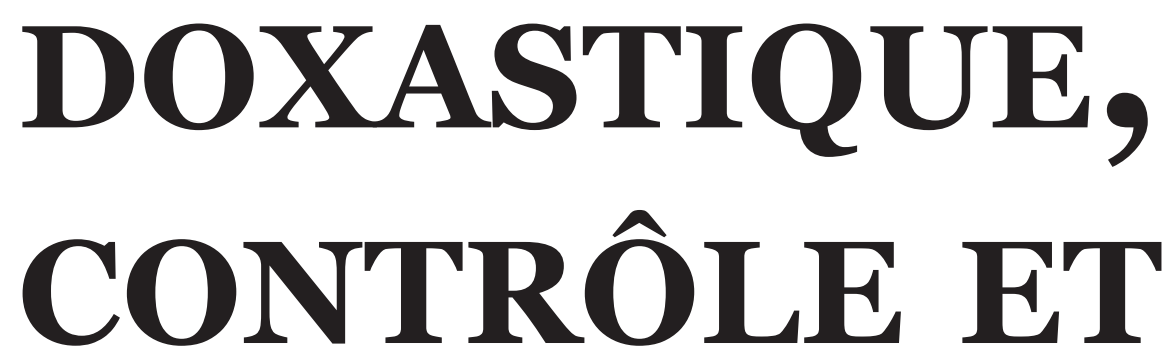

EXPRESSIVITÉ

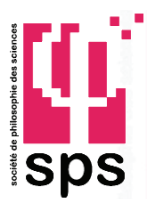

SOCIÉTÉ DE PHILOSOPHIE DES SCIENCES (SPS) 


\section{Aliénation doxastique, contrôle et EXPRESSIVITÉ}

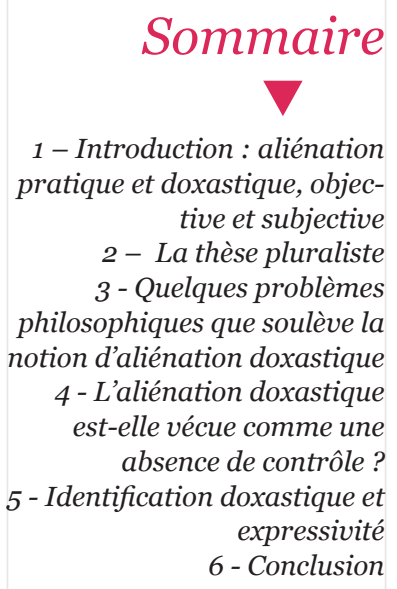

1 - Introduction : aliénation pratique et doxastique, objective et subjective

Une vaste littérature est consacrée aux situations au cours desquelles un agent ne s'identifie pas à un désir qui catalyse pourtant l'une ou plusieurs de ses actions. Selon Frankfurt (1971), il existe alors un conflit ou un décalage au sein de l'agent entre ce désir de premier niveau et une volition de second niveau. L'exemple de Frankfurt est fameux : le « toxicomane malgré lui » est habité par des désirs de premier niveau opposés, à savoir le désir de se droguer et celui de ne pas se droguer, mais il désire simultanément ne plus désirer se droguer. Ce désir de second ordre est précisément ce qui le distingue du toxicomane irréflexif, qui peut parfaitement être animé de désirs conflictuels tout en étant neutre quant à la question de savoir lequel de ses désirs est le plus désirable. Le concept de "volition » est mobilisé dans un tel contexte afin de caractériser les cas où le désir de second niveau ne porte pas simplement sur la possession ou la non-possession de tel désir mais sur sa dimension motivationnelle : le toxicomane malgré lui ne désire pas faire l'expérience du désir de cesser de se droguer afin, par exemple, de voir temporairement le monde sous la perspective de ce désir ou, pour reprendre l'expression du titre de l'article de Nagel (1974), de savoir « quel effet cela fait » de désirer ne pas consommer de drogue. Il désire, au contraire, que ce désir de second ordre se réalise, motive sa conduite et par conséquent produise à terme la cessation effective de l'activité de se droguer. Il aspire ainsi, pour reprendre les termes de Frankfurt, à ce que ce désir constitue sa « volonté ». Du fait de cette distance à soi, l'agent se sent comme étranger à son désir de premier niveau, et ce quelle que soit son intensité, mais également à l'action qu'il catalyse. D’où l'idée selon laquelle ce désir peut être qualifié d' « aliéné » : quoi qu'advenant en moi, il ne serait pas réellement de moi et ne pourrait dès lors être considéré comme pleinement « mien ${ }^{1}$. Le toxicomane malgré lui s'identifie ainsi, du fait de sa volition de second niveau, au désir de premier niveau de ne plus se droguer et se l'approprie plus intimement (Frankfurt 1971, p. 13).

On peut définir la situation du toxicomane comme un cas d'aliénation à la fois pratique et subjective. D'une part, il s'agit d'un cas d'aliénation pratique au sens où les désirs 


\section{Aliénation doxastique, CONTRÔLE ET EXPRESSIVITÉ}

décrits comme aliénés ont un rapport essentiel à l'action. Ultimement, l'objet de ces niveaux de désirs consiste bien, en effet, en l'accomplissement ou non de certains actes - ici, en l'occurrence, le fait de se droguer ou non. Remarquons, cependant, que ceci n'est pas vrai de tout désir. Le désir se distingue en effet de l'intention en ce que les conditions de satisfaction d'une intention, contrairement à celles du désir, sont nécessairement rétro-référentielles : elles n'incluent pas simplement le fait qu'un état de chose advienne mais bien qu'il soit causé d'une manière appropriée ${ }^{2}$ par l'intention en question (Searle 1985, p. 105-106). Privé de la nécessité de cette rétro-référence causale, le désir est indifférent au mode de production de l'état de chose sur lequel il porte. Tout désir occasionnant ce type d'étrangeté à soi ne constitue dès lors pas nécessairement une forme d'aliénation pratique : le fait, par exemple, de désirer qu'il arrive du mal - par opposition au désir de faire du mal - à telle personne tout en désirant simultanément ne pas le désirer ne saurait à proprement parler manifester un tel type d'aliénation.

D'autre part, cette forme d'aliénation peut être décrite comme subjective au sens où elle épouse le point de vue de l'agent. Le désir est en effet vécu par l'individu comme étant en décalage avec la manière dont il évalue et hiérarchise ses désirs. Il paraît en ce sens essentiel au concept d' « aliénation subjective » ainsi entendu que l'agent reconnaisse le contraste entre ce qu'il désire d'une part et ce qu'il estime devoir désirer d'autre part. Dans ce type de situation, ce que Watson (2008, p. 170) nomme le « système motivationnel » de l'agent, c'est-à-dire l'ensemble des considérations qui motivent sa conduite, ne coïncide pas avec son " système évaluatif », à savoir l'ensemble des considérations auxquelles il accorde une valeur. La valeur d'un désir peut être indépendante tant de sa force motivationnelle que de l'intensité avec laquelle il est ressenti puisqu'il est possible que la force d'un désir « ne reflète pas adéquatement le degré auquel on estime son objet » (Watson 2008, p. 162). Par opposition, attribuer à un agent un désir « objectivement » aliéné consisterait à affirmer non pas que son désir efficient de premier niveau ne se conforme pas à la manière dont il hiérarchise ses désirs mais plutôt que son désir va à l'encontre de ce qu'il devrait objectivement désirer - et ce, quel que soit le rapport qu'il entretient à ses états conatifs. Une telle imputation pourrait ainsi consister à évaluer les désirs des agents à l'aune de ce que Williams (1979) nomme des " raisons externes ", c'est-à-dire ici des raisons extérieures tant au système motivationnel de l'agent qu'à son système évaluatif et qui ne peuvent, par principe, pas même figurer parmi les raisons auxquelles il aurait accès s'il délibérait. Ce type d'attribution reviendrait dans certains cas à dire, indépendamment du point de vue de l'agent, que ce désir va à l'encontre de son ou de ses intérêts réels ou " profonds » (Pettit 2004). Une telle conception de l'aliénation a pu être critiquée : en quoi consistent de tels intérêts objectifs ? Quels sont les critères d'identification de ces intérêts censés s'imposer à nous et comment l'attribution de désirs aliénés aux agents pourrait-elle éviter l'arbitraire (Ogien 2013, p. 8990) ? L'évaluation de cette conception de l'aliénation n'entre toutefois pas dans le cadre de cet article. Nous nous focaliserons ici sur l'aliénation subjective ${ }^{3}$.

Ce type de distance à soi ne paraît pas confiné aux états conatifs mais peut, entre autres, caractériser des états affectifs. Certaines émotions semblent en effet pouvoir être décrites comme " aliénées ». Imaginons que je ressente une forte admiration pour une personne et que j'appréhende simultanément cette même admiration comme étant en décalage avec ce que je considère être en général digne d'admiration. Il existe bien, dans ce type de situation, un contraste entre un état émotionnel d'une part et mon évaluation de cet état ainsi que, éventuellement, les émotions de second niveau ${ }^{4}$ que peut susciter cette évaluation d'autre part. Ici encore, je ne peux pleinement m'identifier à ces contenus mentaux. S'ils sont « miens », quoi qu'en un sens faible, puisque c'est bien moi qu'ils affectent, ils ne sont pas pleinement de moi. Je peux certes les anticiper, tenter de les contourner ou de neutraliser leurs effets, à la manière dont Ulysse prédit son propre comportement affectif face aux sirènes et restreint, de ce fait, intentionnellement le champ de ses actions physiquement possibles (Elster 1986). Mais je me sens étranger à eux, comme s'ils constituaient un donné extérieur qui s'impose à moi.

La question qui nous intéresse dans le cadre de cet article porte sur la possibilité d'un équivalent doxastique à cette forme d'aliénation subjective. Le concept de « croyance aliénée » que propose Hunter (2011) est précisément censé nous permettre de décrire les situations au cours desquelles un agent ne s'identifie pas à une croyance qu'il entretient pourtant et ne peut pas ne pas s'attribuer ${ }^{5}$. Il ne s'agit pas ici d'une pensée passagère que je ressens comme extérieure à moi mais d'une croyance au sens strict, autrement dit d'un contenu auquel j'adhère ${ }^{6}$. Plus précisément, l'aliénation doxastique

2 - Cette précision vise à exclure la causalité déviante (Searle 1985, p. 136-139) qui peut advenir entre l'intention et l'état de chose final.

3 - Nous ne parlerons donc pas ici de l'aliénation au sens pathologique ou clinique. Remarquons, de plus, que cette distinction ne renvoie pas à celle que l'on peut trouver chez Marx, dans Les Manuscrits de 1844, entre l'aliénation subjective, entendue comme l'état d'une subjectivité dépossédée de certaines capacités vitales, et l'aliénation objective, à savoir l'état d'une société au sein de laquelle une instance s'autonomise (Haber 20o7) puisque les deux termes caractérisent ici des états mentaux individuels.

4 - La honte d'être admiratif constituerait, dans cette situation, une émotion de second niveau possible. Une telle attitude négative à l'égard d'un état affectif peut favoriser ce qu'Elster (2003) nomme une «transmutation des émotions ». Un sentiment aliéné d'envie peut ainsi faciliter la transformation progressive de l'envie en indignation vertueuse.

5 - Par souci de simplicité, nous ne traiterons pas de la possibilité qu'un agent ressente étrangère une croyance qu'il considère faussement comme sienne. Nous partirons, autrement dit, du principe que l'auto-imputation de la croyance aliénée que $P$ est vraie, au sens où il est vrai qu'il croit que P. Nous examinerons cependant, dans notre dernière partie, la possibilité de croire faussement que la croyance que P est aliénée.

6 - Williams (1991, p. 200) décrit, par exemple, la situation d'un homme qui affirme au cours d'une discussion sur la manière de traiter ses concurrents en politique ou en affaires : "Bien sûr, nous pourrions les faire tuer, mais nous devrions écarter cette éventualité dès le départ ». On peut aisément imaginer le sentiment d'aliénation que peut susciter ce genre d'idées. Pour une analyse de ce cas de figure, voir (Gibert 2009). 


\section{Aliénation doxastique, CONTRÔLE ET EXPRESSIVITÉ}

caractérise le rapport de non-identification qu'un agent entretient à une croyance qu'il sait être " sienne ", au moins en un sens faible, mais qu'il estime simultanément ne pas devoir entretenir. Précisons que cette définition minimale implique que la croyance en question soit consciemment entretenue pour que le conflit en question soit reconnu : une croyance qui se manifesterait, par exemple, par certains comportements tout en n'étant jamais l'objet de jugement ne pourrait pas, selon cette définition, être considérée comme aliénée. Reprenons, à présent, l'exemple d'aliénation doxastique que propose Hunter (2011, p. 224-225). Imaginons une mère qui constate le comportement impulsif et imprévisible de son fils, apprend que son meilleur ami est en prison pour trafic de stupéfiants et reçoit certains appels étranges en plein milieu de la nuit. Sur la base de ces données, elle forme la croyance selon laquelle son fils se drogue. Parce que sa croyance s'ajuste aux données, son comportement doxastique est rationnel et reconnu comme tel. Elle peut pourtant, même dans ce cas de figure, ne pas s'identifier à ce qu'elle croit.

Comment décrire son état d'esprit ? Nous pouvons indifféremment considérer la méta-attitude comme un désir ou une croyance. On peut dire, en première approximation, qu'elle croit que $\mathrm{P}$ (que son fils se drogue) mais désire simultanément ne pas croire que $P$ et/ou croit qu'elle ne devrait pas croire que P. Dans un tel contexte, la différence entre négation interne et négation externe (Elster 1990) n'est pas essentielle : la mère peut indifféremment désirer ne pas croire que $\mathrm{P}$ (par exemple, ne pas même penser à l'éventualité que son fils se drogue) ou désirer croire que non-P, c'est-à-dire penser qu'il ne se drogue pas. Toutefois, précise Hunter, il ne s'agit pas seulement ici - même si ces éléments ne sont pas incompatibles avec la croyance aliénée - de dire qu'elle désire que $\mathrm{P}$ ne soit pas le cas ou qu'elle préférerait ignorer que $\mathrm{P}$ ou les données en faveur de $\mathrm{P}$ (Hunter 2011, p. 224). Au contraire, elle désire ne pas croire que $\mathrm{P}$ au sens où elle désire être le type de mère qui ne croit pas cela de son fils et ce, quelles que soient les données. C'est précisément la rationalité épistémique de son comportement doxastique qu'elle estime blâmable et qu'elle ressent comme une faiblesse, une absence de loyauté eu égard aux devoirs que lui dicte son statut de mère. En d'autres termes, elle ne pense pas qu'elle devrait nécessairement être sourde aux données ou les contourner, en prenant ses désirs pour des réalités ou par duperie de soi. Elle considère plutôt qu'elle devrait penser que les faits défient les données. De son point de vue, cette surdité consciente à son environnement épistémique accroîtrait la valeur de la croyance désirée, comme si une mère aimante ne pouvait pas, quelles que soient les informations dont elle dispose, penser ce genre de choses de son fils.

\section{2 - La thèse pluraliste}

L'une des originalités du propos de Hunter réside dans ce que nous nommerons sa « thèse pluraliste » (TP) selon laquelle les motifs potentiels d'aliénation doxastique sont pluriels. Je peux ainsi, selon TP, croire que je crois que $\mathrm{P}$ et simultanément croire que je ne devrais pas croire que $\mathrm{P}$ pour des raisons :

- épistémiques (lorsque je crois que ma croyance va à l'encontre des données auxquelles j'ai accès et/ou est en contradiction avec certaines de mes autres croyances ${ }^{7}$ ) ;

- pragmatiques (si je considère, par exemple, que ma croyance que $\mathrm{P}$ suscitera des effets non désirés) ;

- morales (si je crois que le fait d'entretenir la croyance que $\mathrm{P}$ va à l'encontre de certaines de mes valeurs morales $^{8}$ ).

Si TP est vrai, ces motifs d'aliénation doxastique peuvent alors être combinés. On peut, par exemple, concevoir des raisons d'ordre pragmatico-morales ${ }^{9}$ ou pragmatico-épistémiques d'aliénation doxastique. Illustrons cette dernière idée. Je peux fort bien croire que $\mathrm{P}$ en basant ma croyance sur des raisons épistémiques et croire que je ne devrais néanmoins pas croire que $P$ pour des raisons pragmatiques dont la finalité est épistémique. Reprenons une expérience de pensée proposée par Railton (1994, p. 84). Imaginons qu'il existe, eu égard au travail cognitif, un mécanisme analogue à la fameuse " main invisible » d'Adam Smith en économie, à savoir une forme d'esprit invisible faisant en sorte que c'est uniquement en adhérant à des croyances conformes à ce qu'ils ont institutionnellement intérêt à croire et en cherchant à défendre leurs croyances d'une manière épistémiquement " impure », par exemple en collectant de manière biaisée les données, que les scientifiques de diverses écoles contribueraient au progrès global des sciences et à la maximisation des vérités. Admettons que tel chercheur prenne conscience de cette loi.

7 - Nous ne distinguerons pas ici l'aliénation épistémique de l'aliénation évidentielle, à la manière dont Owens (2002) distingue l'akrasia épistémique de l'akrasia évidentielle. Pour illustrer l'idée d'aliénation doxastique épistémiquement motivée, Hunter (2011, p. 223) imagine, entre autres, Descartes ne parvenant pas à réellement croire que les vérités mathématiques auraient pu être autres qu'elles ne sont alors qu’il considère simultanément avoir les meilleures raisons du monde de penser que Dieu est omnipotent à ce sujet.

8 - Le déontologisme et le conséquentialisme fournissent deux sources différentes d'aliénation doxastique pour des motifs moraux. Je peux considérer que ma croyance ne respecte pas une règle d'action morale et, ainsi, que le simple fait de croire que P est intrinsèquement immoral (et ce quel que soit l'état du monde). Mais je peux aussi ressentir comme étrangère à moi-même une croyance car il me semble que le fait de l'entretenir produira des effets qui iront à l'encontre de la promotion du plus grand bien possible dans le monde. Le problème est toutefois de savoir si une croyance peut être ainsi soumise au même type d'évaluation morale qu'une action.

9 - Les motifs pragmatico-moraux d'aliénation peuvent être pensés sur le modèle de l'éthique de responsabilité wébérienne (Weber 1959) : je peux ressentir comme aliénée une attitude qui, quoi que manifestant ou incarnant mon adhésion axiologique, sera perçue comme allant à l'encontre de sa réalisation effective, c'est-à- 


\section{Aliénation doxastique, CONTRÔLE ET EXPRESSIVITÉ}

Comment ce dernier se représentera-t-il ce qu'il doit croire ? En un sens pragmatico-épistémique, il aurait une bonne raison de persister à croire en sa théorie puisque cette croyance est censée produire indirectement des effets épistémiques optimaux. Toutefois, s'il perçoit les insuffisances de cette théorie et la part, par exemple, de rationalisation d'intérêt institutionnel qu'elle comporte, il a également des raisons épistémiques de réviser au moins son degré de croyance initial. Quittons la lettre du texte et imaginons que sa vie entière soit focalisée sur l'objectif de contribuer à la maximisation des connaissances et qu'il se rende simultanément compte de son incapacité, bien compréhensible, à réviser sa croyance sur la base de telles considérations instrumentales, fussent-elles à finalité épistémique. Notre chercheur peut alors persister à croire que $\mathrm{P}$ pour des raisons épistémiques et se sentir extérieur à cette croyance parce qu'elle lui semble aller à l'encontre de la valeur selon lui la plus haute. Il estime, autrement dit, qu'il devrait ajuster son état doxastique à ces raisons pragmatico-épistémiques.

Définissons ainsi TP :

(TP) Les motifs d'aliénation doxastiques sont pluriels (et donc pas seulement intrinsèquement épistémiques)

TP a au moins deux conséquences intéressantes. Tout d'abord, si l'on définit, de manière minimale, l'akrasia ou incontinence épistémique comme la coexistence de la croyance que $\mathrm{P}$ et du jugement de second niveau selon lequel je ne devrais pas croire que $\mathrm{P}$ parce que je ne suis pas épistémiquement justifié à croire en la vérité de $\mathrm{P}$ (Engel 2007 ; Hookway 2001; Ribeiro 2011) ${ }^{10}$, alors l'aliénation doxastique n'est pas réductible à l'incontinence épistémique. Dès lors, et de manière plus fortement contre-intuitive, l'incontinence épistémique n'est pas nécessairement une source d'aliénation doxastique. Dit autrement, je peux parfaitement m'identifier à une croyance dont je suis pourtant conscient de l'irrationalité.

Il m'est ainsi possible, par exemple, de croire simultanément que mon ami est innocent, que l'hypothèse de sa culpabilité est plus vraisemblable que celle de son innocence et néanmoins de ne pas me sentir étranger à cette croyance manifestement contradictoire avec les données parce que l'entretenir me semble conforme au type de loyauté que l'amitié me dicte à son égard. Si l'on suit les analyses de Stroud (2006), être ami avec $\mathrm{X}$ inclut en effet, à titre d'élément constitutif, un certain engagement à la partialité épistémique : l'exigence de loyauté à son égard prescrirait ainsi, dans certains contextes, un rapport sélectif aux données, autrement dit un biais favorable à $\mathrm{X}$, tant dans la méthode de formation de ma croyance concernant $\mathrm{X}$ que dans la conclusion de ce processus, c'est-à-dire dans la croyance qui en résulte (Stroud 2006, p. 504-509). Inversons, dès lors, la situation. Imaginons que je ne par- vienne pas, malgré la conscience que j'ai de mon obligation morale à la partialité épistémique, à ne pas ajuster de manière impartiale mes croyances aux données. J'ai beau tenter, par exemple, de mobiliser systématiquement des hypothèses alternatives à ce que je désire ne pas croire de $\mathrm{X}$ ou d'exercer mon esprit critique de manière asymétrique à l'égard des données, je ne parviens pas réellement à mettre en œuvre cette méthode ni dès lors à produire les effets escomptés sur moi tant je suis convaincu de la culpabilité de mon ami sur la base des données initiales. Ma croyance en la culpabilité de $\mathrm{X}$ s'ajuste parfaitement aux données disponibles et, en cela, ma pratique épistémique est rigoureusement la même que s'il s'agissait d'évaluer les actions d'un parfait inconnu. Je peux alors avoir honte de ce que je crois du fait de l'absence de loyauté que mon impartialité épistémique traduit et ressentir ainsi comme étrangère une croyance que je sais pourtant être épistémiquement irréprochable. Dans les deux cas, l'appréciation de mon état doxastique en termes de loyauté supplante son évaluation épistémique : l'identification à mon état outrepasse son inadéquation évidentielle dans le premier cas ; dans le second, l'aliénation doxastique réside précisément dans mon incapacité à ajuster ma croyance à cette appréciation.

\section{3 - Quelques problèmes phi- losophiques que soulève la no- tion d'aliénation doxastique}

Le concept ainsi défini d'aliénation doxastique n'est pas sans poser un certain nombre de problèmes. Une première difficulté vient de la conjonction, dans l'article de Hunter, de TP avec ce que nous nommerons la « thèse normative » $\mathrm{TN}$, que nous formulerons ainsi :

(TN) L'aliénation doxastique manifeste un désordre cognitif intrinsèque

Selon TN, l'absence d'identification à l'égard de nos propres états doxastiques refléterait nécessairement une défaillance interne à l'exercice de nos facultés intellectuelles (Hunter 2011, p. 236). On pourrait en effet, à première vue, interpréter la formation de la méta-attitude en question comme exprimant une forme de vice épistémique (Pouivet 2008) : n'est-il pas intellectuellement blâmable d'évaluer négativement sa croyance à l'aune de motifs non-épistémiques ? L'un des aspects originaux du propos de Hunter tient cependant au fait que cette défaillance porte, selon lui, sur la croyance de premier niveau. C'est bien le fait de ne pas pouvoir ajuster ce que l'on croit à ce que l'on estime devoir croire qui pose problème. $\mathrm{TP}$, pris isolément, n'a pas cette conséquence normative : je peux ainsi ne pas m'identifier, pour des raisons morales ou pragmatiques, à l'une de mes croyances sans pour autant en 


\section{Aliénation doxastique, CONTRÔLE ET EXPRESSIVITÉ}

tirer la conclusion que je ne devrais pas l'entretenir, autrement dit que mon comportement doxastique manifeste une forme d'échec. Une question centrale est de savoir si TN est compatible avec TP. Peut-on, autrement dit, affirmer d'une part que les motifs d'aliénation sont pluriels et que, d'autre part, l'aliénation manifeste en tant que telle une défaillance dans le comportement doxastique du sujet ainsi aliéné ? La conjonction de TP et de TN semble nous contraindre à admettre l'existence de bonnes raisons non-épistémiques de croire, ce qui est loin d'être évident ${ }^{11}$. Enfin, quel critère nous permet d'identifier telle situation d'incontinence épistémique comme un cas d'aliénation doxastique?

Nous n'explorerons pas ces réflexions dans la suite de cet article. Je souhaite ici m'interroger sur la définition minimale de l'aliénation doxastique que l'on peut tirer de cette analyse : que signifie, autrement dit, le fait de ne pas s'identifier à telle de nos croyances ? Si nos croyances peuvent être soumises à des standards divers, elles sont susceptibles de générer des méta-attitudes tout aussi diverses. TP semble donc intuitivement correct, que l'on accepte ou non TN. TP nomme en effet une réalité psychologique difficilement récusable : d'une part, le sentiment que certaines de nos croyances consciemment entretenues sont vécues comme extérieures à nous et, d'autre part, le fait que ce sentiment d'extériorité n'est pas nécessairement lié à des motifs épistémiques. Ne pouvons-nous pas, en effet, nous sentir distants de certaines de nos croyances parce qu'elles nous semblent par exemple irrespectueuses, conformistes, contre-productives ou nocives ? Toutefois, comment définir au mieux l'aliénation doxastique ? Est-elle, par exemple, réductible au sentiment d'étrangeté à soi que suscite en nous l'apparition de certaines croyances ? Si tel est le cas, nous serions alors par principe immunisés contre l'erreur en ce domaine, c'est-à-dire quant à la question de savoir si nos croyances sont effectivement aliénées. Doit-on cependant considérer que parce qu'il s'agit d'une forme d'aliénation subjective, il est par principe impossible de croire faussement entretenir une croyance aliénée?

Hunter caractérise le vécu d'aliénation doxastique de plusieurs manières ${ }^{12}$. Nous nous intéresserons à deux aspects très suggestifs, mais peu explicités, de son analyse, à savoir le fait que le sujet victime d'aliénation doxastique vit ou appréhende sa croyance comme :

(1) le signe d'une défaillance en termes de contrôle sur ses états doxastiques - ce que nous appellerons « la thèse du contrôle »
(2) étant inexpressive de ce qu'il nomme son « moi véritable » ou «profond » («true or deepest self») - ce que nous nommerons la « thèse de l'expressivité »

Cette double caractérisation se retrouve dans certaines expressions : dire que l'on croit quelque chose «malgré soi » ou « à son insu » peut désigner une perte de contrôle mais également le fait que la croyance en question ne nous semble pas refléter adéquatement ce que nous sommes. Quoi que les thèses (1) et (2) soient présentées par Hunter comme substituables, il semble à première vue nécessaire de les dissocier. On peut certes penser que le contrôle est expressif de notre personne au sens où en contrôlant ce que nous pensons, nous en serions plus pleinement l'auteur. Toutefois, le « moi » ne s'exprime-t-il pas pleinement lorsqu'il se manifeste spontanément, autrement dit le contrôle n'introduit-il pas une forme d'inhibition dont la source peut s'avérer extérieure à l'agent ? À aucun moment, cependant, Hunter n'explicite réellement le contenu de ces deux idées : que peut bien signifier le fait d'appréhender sa croyance comme étant hors de contrôle ou bien comme n'exprimant pas pleinement ce que nous sommes?

Ma visée est double. Je tente, dans un premier temps, de montrer que la thèse (1) ne décrit pas correctement le vécu d'aliénation. En d'autres termes, il me semble peu vraisemblable de considérer que l'individu aliéné vit sa propre croyance comme une défaillance en termes de " contrôle doxastique ", et ce quel que soit le sens que l'on donne à cette expression. Dans un second temps, après avoir admis que la thèse (2) est plausible d'un point de vue psychologique, je m'interroge sur la question de savoir en quel sens elle est susceptible de fournir un cadre conceptuel adéquat au phénomène de l'aliénation doxastique. Ma question directrice, qui excède le propos de Hunter, est alors la suivante : quelle est la valeur épistémique de l'appréhension ou du "vécu » d'inexpressivité décrit en (2) ? Plus précisément, le concept d'aliénation doxastique subjective suppose-t-il que nous soyons immunisés contre l'erreur en ce domaine ? Cette interrogation me conduit à interroger les critères d'identification de l'expressivité de nos croyances, à souligner le décalage possible entre l'inexpressivité vécue et l'inexpressivité réelle d'un état doxastique et ainsi à esquisser une conception de l'aliénation doxastique subjective qui prenne en compte cette possibilité. proposer une approche originale de la distance que l'on peut entretenir à l'égard de nos croyances.

12 - Nous ne traiterons pas ici de l'un des critères d’identification, indépendant des idées de contrôle et d'expressivité, de l'aliénation doxastique que Hunter considère comme décisif, à savoir l'accès épistémique particulier que nous aurions à nos croyances aliénées : ces dernières seraient, tout comme les croyances d'autrui, essentiellement accessibles par observation (Hunter 2011, p. 238-239). 


\section{Aliénation doxastique, CONTRÔLE ET EXPRESSIVITÉ}

\section{4- L'aliénation doxastique est- elle vécue comme une absence de contrôle?}

Reprenons l'exemple de Hunter. La mère fait l'expérience d'un décalage entre son état doxastique et une attitude de niveau supérieur qui le prend pour objet : elle aimerait que sa croyance soit conforme à son amour maternel et à ce qu'elle se représente comme étant une forme de loyauté à l'égard de son fils. Fait-elle, pour autant, l'expérience d'une absence de contrôle doxastique ? Pour que cette situation soit vécue comme une absence d'emprise sur nos croyances, il faudrait qu'aux yeux du sujet une telle emprise ait un sens et paraisse au moins correspondre à une réalité dont il fait parfois l'expérience. En quel sens l'aliénation doxastique peut-elle inclure une telle expérience ? À quelle étape du processus de formation de croyance cette absence de contrôle se ferait-elle sentir ? Notre examen doit porter, d'une part sur l'identification du type de contrôle standard que nous aurions sur nos croyances, contrôle qui devrait nous permettre de mieux appréhender l'identification doxastique et à l'aune duquel, d'autre part, les cas d'aliénation pourraient être identifiés et décrits comme tels ${ }^{13}$. Le propos de Hunter n'est pas sans ambiguïté car le sentiment d'absence de contrôle qui accompagne les croyances aliénées est censé porter, selon lui, soit sur l'exercice de nos facultés cognitives soit sur leurs produits (Hunter 2011, p. 221), ce qui correspond à des modes de compréhension distincts du contrôle doxastique. Ajoutons enfin que, conformément à TP, ce type de contrôle doit pouvoir être soumis à des motifs divers, autrement dit également à des motifs non-épistémiques.

\subsection{L'aliénation doxastique est-elle vécue} comme un échec à exercer un contrôle direct ou indirect sur nos croyances?

Une première interprétation possible du contrôle doxastique correspond à la thèse du volontarisme doxastique direct qui consiste à affirmer que nous pouvons croire " à volonté ", c'est-à-dire croire que $\mathrm{P}$ parce que nous le décidons. Étant donné la diversité des raisons pour lesquelles nous pouvons former des intentions, cette thèse semble compatible avec TP. De plus, c'est une manière plausible d'interpréter l'idée de Hunter selon laquelle le sujet aliéné fait l'expérience d'une incapacité à contrôler le produit de ses opérations cognitives, à savoir la croyance elle-même. Peut-on, cependant, transposer au niveau doxastique la différence entre ce qui advient en moi et ce que je fais ? Si tel est le cas, une croyance peut découler d'une intention de croire au sens où une action découle d'une intention d'agir. L'individu aurait dès lors une prise immédiate sur ses propres adhésions. Soulignons que l'immédiateté dont il est ici question est motivationnelle et non ontologique (Bennett 1990, p. 89) : le fait que je puisse lever le bras suite à ma décision de le faire, et donc sans accomplir d'actions intermédiaires, n'implique nullement qu'aucune séquence physiologique ne soit causalement intervenue entre ces deux événements.

Un tel contrôle semble toutefois impossible. Remarquons, tout d'abord, que nous n'expérimentons pas de contrôle direct de cette sorte sur nos croyances : nous ne faisons en effet pas ordinairement l'expérience d'une consécution entre une décision de croire et l'émergence d'une croyance conforme à cette décision au sens où nous faisons l'expérience du lien entre la décision de lever le bras et l'action de le lever. Ensuite, comme l'a montré Williams (1973, p. 148-151), le fait de croire que $\mathrm{P}$ est incompatible avec la croyance selon laquelle la croyance que $P$ résulte de ma décision antérieure de croire que $\mathrm{P}$. Le fait de considérer que ma croyance que $\mathrm{P}$ provient de mon intention de croire que $\mathrm{P}$ la relativise en effet à mes propres yeux et ainsi la désactive. La croyance est par nature un état qui vise la vérité, et ce quels que soient les désirs et intentions du sujet qui l'entretient, au sens où le sujet doit se représenter sa croyance comme visant le vrai et non l'agréable, l'utile, etc. Or savoir que je crois que P parce que je l'ai décidé implique d'avoir conscience du fait que ma croyance résulte d'un processus qui n'est pas de nature à fournir une raison épistémique de croire que $\mathrm{P}$, autrement dit une raison de croire en la vérité du contenu $\mathrm{P}^{14}$.

Si certains aspects de l'analyse de Williams ont été contestés (Bennett 1990 ; Setiya 2008), ces critiques ne paraissent pas entamer le cœur de son argument qui consiste à affirmer que la formation de la croyance ne peut, par principe, constituer une action intentionnelle (Setiya 2008, p. 44-45). Si, en effet, le concept d' « action » inclut une forme de " connaissance pratique ${ }^{15}$ (Anscombe 2002, p. 150-151), alors l'idée de formation intentionnelle de croyance est contradictoire avec les contraintes épistémiques qui pèsent sur le concept de croyance. Former intentionnellement la croyance que $P$ implique la conscience, ou à tout le moins la croyance (Setiya 2008, p. 45-47), que je suis en train de former intentionnellement la croyance que P. Lors de la formation intentionnelle de la croyance, le sujet faisant advenir intentionnellement

13 - Il est certes possible de dissocier la question du contrôle doxastique effectif de celle du sentiment de contrôle doxastique : un sujet pourrait croire de manière erronée qu'il contrôle ordinairement ses croyances et ressentir dès lors les cas d'aliénation comme spécifiques, ce que notre première sous-partie tentera de réfuter. Inversement, il peut contrôler ordinairement ses croyances sans le savoir et ne pas se rendre compte de la spécificité des cas d'aliénation - ce qui nous semble assez peu plausible. Il nous a paru plus vraisemblable de partir de l'idée selon laquelle le sentiment de perte de contrôle devait être éprouvé à l'aune d'une expérience véridique de contrôle.

14 - Un autre argument consiste à dire que les cas de causalité déviante doxastique diffèrent des situations de causalité déviante décrites en philosophie de l'action en ce que nous ne faisons précisément pas l'expérience, dans le premier cas, d'une perte de contrôle doxastique (Buckareff 20o8). D’un point de vue phénoménologique, les cas de rationalisation et de non-rationalisation de la croyance que P par telle raison $R$ sont en effet indiscernables. Pour une analyse de la causalité déviante interne aux processus de formation de croyances, voir (Kelly 2002).

15 - Cette expression renvoie au fait qu'effectuer une action enveloppe la connaissance non-observationnelle et non-inférentielle du fait qu'on effectue cette même action. 


\section{Aliénation doxastique, CONTRÔLE ET EXPRESSIVITÉ}

cet état doxastique entretiendrait dès lors deux croyances contradictoires : il croirait en effet que P (puisque la croyance est censée s'installer en lui) et ne croirait pas que $\mathrm{P}$ (puisque c'est précisément parce qu'il a conscience de ne pas croire que $\mathrm{P}$ qu'il forme et satisfait l'intention de croire que P). Que peut bien signifier le fait de réaliser cette intention? Autrement dit, en quoi peut bien consister la formation intentionnelle de la croyance, si cette supposée « action » est censée inclure la conscience du fait que l'état doxastique qu'il installe procède de sa seule intention de croire que $\mathrm{P}$ ? La formation intentionnelle de la croyance serait soumise à des standards de justification ici contradictoires, à savoir épistémiques et pragmatiques $^{16}$. Il lui faudrait dès lors oublier cette intention au moment même où il forme intentionnellement la croyance en question. Toutefois, une telle ignorance de la production intentionnelle de la croyance va précisément à l'encontre $\mathrm{du}$ réquisit de connaissance pratique contenu dans notre concept d'action.

On comprend pourquoi, pour des raisons conceptuelles, il semble non pas, certes, psychologiquement impossible mais tout de même assez peu vraisemblable d'imaginer des êtres en proie à un sentiment d'aliénation doxastique du fait que leur décision de croire échoue. Ce sentiment n'est pas absurde au sens, seulement, où l'on saurait que cette production immédiate est empiriquement impossible, un peu comme le serait l'avènement de l'oubli à partir de la décision d'oublier. Mais il se révèle également incompatible avec notre concept de croyance puisque, comme nous l'avons vu, la rétro-référentialité de l'intention implique qu'il entre dans les conditions de satisfaction de cette attitude mentale que l'état réalisé soit adéquatement causé par l'intention elle-même. Or, ces conditions ne peuvent pas être considérées par l'individu doxastiquement aliéné comme réalisables du fait de la nature de l'état qui vise à être produit, autrement dit du fait de notre concept de croyance et de sa liaison constitutive avec celui de vérité. Le vécu d'aliénation doxastique ne peut donc être décrit comme un sentiment d'incapacité à exercer un tel type de contrôle $\mathrm{e}^{17}$.

Une seconde interprétation de la thèse du contrôle consisterait à dire que le sujet aliéné fait l'expérience d'une incapacité à exercer un contrôle indirect sur ses croyances. L'aliénation doxastique inclut-elle le sentiment d'une telle défaillance?
L'idée de « contrôle doxastique indirect » renvoie parfois à des formes de contrôles non-épistémiquement orientées : dans ce cas, la croyance à laquelle le sujet aspire est désirée pour des propriétés autres que la vérité ou la rationalité de son contenu. Certains processus de ce type incluent des médiations épistémiques, d'autres en sont dépourvus ${ }^{18}$ : l'exemple des croyances obtenues par hypnose (Williams 1973, p. 150) constitue un cas paradigmatique de production doxastique n'incluant aucune médiation épistémique. Considérons toutefois une autre forme d'auto-conditionnement : l'exposition sélective à certaines données en faveur de l'advenue d'une croyance désirée. Si un tel processus de formation de croyances paraît bien épistémiquement irrationnel, il s'adosse toutefois à des médiations épistémiques puisqu'il s'agit de se laisser affecter par des données, certes préalablement triées ${ }^{19}$. Serions-nous parvenus ici à reconnaître un cas standard de contrôle à l'aune duquel identifier l'aliénation doxastique?

Il serait étonnant de considérer cette action instrumentale sur soi comme une source d'identification. Se manipuler, n'est-ce pas précisément agir sur ses croyances comme si elles appartenaient à d'autres et constituaient, en quelque sorte, un donné extérieur sur lequel je tente d'influer ? Je peux, par exemple, agir de la même manière sur mes sensations, c'està-dire me les infliger. Dira-t-on, dans ce cas, que je m'identifie à mes sensations parce que je les cause en moi (Moran 2013, p. 194) ? Certes, la mère, dans l'exemple de Hunter, pourrait s'identifier à une croyance obtenue de la sorte. Mais est-ce la défaillance de ce type de procédé que Hunter considère comme un échec en termes de contrôle ? Que ces stratagèmes puissent éventuellement remédier à une situation d'aliénation ne signifie pas que le vécu d'aliénation inclue le sentiment de leur échec. Il semble, autrement dit, absurde de penser le vécu d'aliénation doxastique comme la conscience d'une incapacité à effectuer une telle auto-manipulation.

\subsection{L'aliénation doxastique est-elle vécue comme un défaut d' « agentivité »?}

Existe-t-il une forme d'activité doxastique qui ne se confonde pas avec les types d'auto-affection précédemment étudiés et qui nous permettre de comprendre le vécu d'aliénation? Moran (2002) a tenté de déconnecter la question de l'activité mentale de celle du contrôle au sens usuel du terme et de faire de l'agentivité ainsi redéfinie le critère de l'identification

16 - Puisqu’il s'agit, d’un côté, de croire que P est vrai et, de l'autre, de croire que P uniquement pour satisfaire lïntention de croire que P, c'est-à-dire pour une raison non-épistémique. En quoi peut dès lors bien consister le fait d'agir sur la base d'une telle intention?

17- Une décision de croire épistémiquement motivée ne fournit-elle pas, dès lors, un exemple parfait de contrôle doxastique ? La réfutation du volontarisme doxastique direct ne repose cependant pas sur l'opposition entre les raisons épistémiques de croire et les raisons non-épistémiques de vouloir-croire car la fausseté de cette thèse est également perceptible lorsque les raisons de vouloir-croire ont une composante intrinsèquement épistémique (c'est-à-dire lorsqu'elles ne sont pas des raisons pragmatiques à finalité épistémique). Je suis incapable de former intentionnellement telle croyance quand bien même cette formation serait épistémiquement rationnelle puisque c'est l'ajustement de la croyance aux données que j’échoue précisément à contrôler. Le seul état mental de ce type qui peut valoir comme une action épistémique au sens strict et qui donc implique un contrôle réel est ce qu'on peut nommer, à partir des analyses de Cohen (1989, p. 24), «l'acceptation épistémique ».

18 - La méthode Coué consiste bien en un processus non-épistémiquement finalisé et peut recevoir deux types d'interprétation. Soit il s'agit de faire en sorte que la pure répétition verbale agisse mécaniquement sur notre esprit, auquel cas aucune médiation épistémique n'est requise. Soit, afin d'opérer, la répétition tend à produire des actes couronnés de succès qui confirment en retour lïmage gratifiante que je tente dinstaller en moi et donc le contenu des énoncés répétés - auquel cas une médiation épistémique intervient.

19 - L'objet sur lequel notre contrôle n'est donc pas ici directement doxastique : je fais intentionnellement en sorte de croire que P. Les actes sont des moyens productifs et non constitutifs de la croyance (Setiya 2008, p. 41) : ils ne constituent pas les parties d'une action globale que l'on pourrait nommer « former la croyance que P ». L'unité téléologique est ainsi brisée avec l’advenue de la croyance. 


\section{Aliénation doxastique, CONTRÔLE ET EXPRESSIVITÉ}

mentale. Que peut bien, toutefois, signifier le fait d'entretenir une telle relation active à ses croyances ? L'échec de ce type de relation explique-t-il le sentiment de passivité censé appartenir à la phénoménologie de l'aliénation doxastique, autrement dit au rapport en première personne qu'entretient l’individu à sa croyance aliénée ?

L'identification à un état mental ne saurait, dans cette perspective, être identique à l'approbation que suscite en nous son contenu. Socrate approuvait les injonctions de la voix du démon. Cette voix demeurait toutefois initialement étrangère à lui, en partie parce qu'il n'avait pas accès aux raisons de ces injonctions (Moran 2002, p. 194). Elle ne peut cependant pas non plus être définie par la combinaison de l'approbation d'une attitude mentale et de l'accessibilité des raisons de l'entretenir car l'identification suppose la capacité d'ajuster nos états mentaux à ces appréciations. Nous ne sommes pas de simples « évaluateurs » de nos attitudes puisque l'évaluation en question a une dimension potentiellement motivationnelle (Pettit et Smith 1996). Le simple fait d'échanger des raisons suppose la reconnaissance de cette aptitude : nous ne nous contentons pas d'extérioriser nos raisons mais tentons d'affecter (ou nous laissons affecter par) les raisons des autres. Nous sommes ainsi bien supposés conformer nos états cognitifs, conatifs ou même affectifs à ce que nous estimons, du fait de ces raisons, devoir croire, désirer ou ressentir.

Cette remarque nous mène, selon Moran, à une propriété essentielle de l'identification. Nous ne pouvons considérer comme pleinement «nôtres » que des états mentaux dont l'exigence de justification est interne, autrement dit des états « judgment-sensitive » (Moran 2002, p. 197). Ces expressions indiquent une double dépendance : les raisons que tel individu a, par exemple, de croire que $\mathrm{P}$ font une différence quant à la justification du contenu de la croyance mais également quant au fait qu'il entretienne actuellement la croyance que $\mathrm{P}$. La perte de ces raisons n'affecte dès lors pas seulement le poids normatif de nos états doxastiques : elle est également censée peser sur leur persistance (Moran 2013, p. 192). Croire que $\mathrm{P}$, c'est certes être disposé à défendre en termes épistémiques la légitimité de cette croyance mais également, entre autres, à réviser son contenu si les raisons en faveur de non-P supplantent les raisons de croire que P. La reconnaissance de la légitimité de ces implications en termes de comportement doxastique témoigne du fait que nous nous considérons comme exerçant une autorité rationnelle au moins potentielle sur nos croyances. Nous assumons, autrement dit, le fait que ces dernières sont censées refléter notre évaluation des raisons et ainsi exprimer notre relation au monde (Moran 2013, p. 92). Lorsque tel est le cas, c'est-à-dire lorsque ma croyance exprime mon appréciation épistémique, elle est pleinement mienne : j'en suis l'auteur et entretient une relation active à son égard. Tel serait le sens de l'agentivité doxastique ${ }^{20}$.

Quel est le lien entre l'agentivité et le contrôle doxastique? Cette conception non-volontariste de l'activité doxastique non seulement diffère de toute forme de contrôle doxastique instrumental mais, en réalité, elle l'exclut. Parce que ma croyance reflète mon évaluation, autrement dit parce qu'elle est censée résulter adéquatement de mon appréciation des raisons, elle ne peut être produite sur un mode extérieur. Je peux certes être responsable de manière externe, et selon les processus indirects précédemment décrits, de ma propre croyance comme je suis susceptible de l'être de celle d'autrui. Je peux même produire en moi, sur un mode instrumental, une croyance extérieurement conforme à mon appréciation des raisons. Imaginons, en effet, un cas de causalité déviante interne à ma personne : par un mécanisme non-rationnel minutieusement élaboré, je parviens à installer progressivement en moi une croyance conforme, non pas simplement aux données, mais bien à mon évaluation initiale des données et peut-être, ainsi, à remédier à une situation akratique. Parce que mes raisons ne produisent pas de la manière appropriée ma croyance, cette situation ne peut toutefois constituer un cas d'agentivité doxastique. Cette dernière implique en effet que j'entretienne telle croyance, non pas seulement en conformité avec mon évaluation des raisons ni simplement du fait de cette évaluation, mais bien pour ces raisons. Le concept de «sensibilité aux raisons » (« responsiveness to reasons ») désigne ce mode de production adéquat ${ }^{21}$.

Je ne peux ainsi, par principe, agir sur les croyances d'autrui comme j'agis sur les miennes quand je suis doxastiquement actif : lorsque j'expose à autrui certaines raisons de croire, je suis incapable de faire en sorte qu'une croyance résulte adéquatement en lui de sa sensibilité au contenu des raisons exposées. Le fait même d'échanger des raisons suppose dès lors l'attribution, à notre interlocuteur, de cette aptitude à l'agentivité (Moran 2002, p. 201). On peut, ici encore, parler de « contrôle » doxastique mais, comme l'explique dans le même esprit Hieronymi (2008, p. 370-371), ce type de contrôle s'effectue en orientant notre regard, non pas sur nos états doxastiques, mais sur le monde. De même que je ne contrôle pas mes intentions en les considérant elles-mêmes mais en orientant mon regard sur les raisons de former telle intention et donc sur leurs objets (p. 368-369), de même je « contrôle » telle croyance en considérant directement les raisons de l'entretenir et donc l'état de chose qu'elle est censée représenter. La tentative de contrôler instrumentalement ma croyance me placerait ipso facto dans une situation d'extériorité face à l'état mental ainsi suscité. 


\section{Aliénation doxastique, CONTRÔLE ET EXPRESSIVITÉ}

Comment comprendre, dès lors, le vécu d'aliénation ? Le sentiment de passivité qui caractérise l'aliénation doxastique découlerait de cette perte d'agentivité : les cas d'aliénation sont des cas de distorsion qui adviennent lorsqu'une croyance survient ou persiste malgré mon appréciation épistémique. En ce sens, ma croyance échoue à refléter mes raisons. Dans ces cas, la conscience de ma croyance est détachée du " sens des raisons qui l'étayent » (Moran 2013, p. 163). D'où l'idée selon laquelle le sujet victime d'aliénation entretient un rapport empirique à son état aliéné : il « constate » qu'il croit que $\mathrm{P}$ malgré le fait que son évaluation des données ou sa délibération est censée déboucher sur la croyance que non$\mathrm{P}^{22}$. Le sentiment d'absence de contrôle exprimerait ainsi la conscience d'un défaut d'ajustement rationnel.

Illustrons cette idée. Imaginons qu'un individu croit que $\mathrm{P}$ du fait de certaines raisons qu'il estime cependant défaites et se trouve ainsi dans l'incapacité de ne pas accorder un poids épistémique disproportionné à telle(s) donnée(s). Deux situations, qui correspondent respectivement aux deux types de défaiseurs possibles, peuvent être envisagées ${ }^{23}$. Il peut, d'une part, avoir affaire à des undercutting defeaters (Pollock 1986) s'il se sent contraint de baser sa croyance sur des données qu'il juge pourtant défaites en tant que données. Par exemple, il ne parvient pas à ne pas croire que $\mathrm{P}$ sur la base de tel témoignage alors que tout indique à ses yeux que le témoin est un menteur pathologique. Mais il peut également, d'autre part, avoir accès à des rebutting defeaters si, par exemple, il dispose non pas d'informations sur le témoin mais de certaines données qui portent directement atteinte, et de manière décisive, à la vérité de $\mathrm{P}$. L'individu épistémiquement akratique ne parviendrait pas ici à neutraliser l'attractivité du témoignage en faveur de P malgré sa conscience de la supériorité de la connexion évidentielle entre les données alternatives et non-P. La thèse de Moran consiste à dire, dans les deux cas, que parce qu'elle est (directement ou non) défaite par son évaluation épistémique globale, la donnée potentielle en faveur de $\mathrm{P}$ que constitue le témoignage ne saurait être, malgré son poids normatif apparent et sa force motivationnelle, considérée comme sa raison. L'individu aurait, dans ces situations, le sentiment de ne pas contrôler ses productions doxastiques et entretiendrait ainsi une croyance aliénée.

Cette conception du rapport entre activité et identification doxastique se heurte toutefois à plusieurs objections. Tout d'abord, une ambiguité demeure dans cette analyse. Les concepts d'aliénation et d'identification sont censés à la fois avoir une réalité subjective et dépendre de la sensibilité ou réactivité adéquate aux raisons. Or, ces deux critères peuvent entrer en conflit. Imaginons la situation suivante :

\section{(1) Je crois que P}

(2) Ma croyance que P est le produit adéquat de mon appréciation des raisons

\section{(3) Je suis de conscient de (2)}

(4) Je crois de manière irrationnelle que mon appréciation est biaisée

Je crois, par exemple, du fait d'un pressentiment fort et injustifiable, que tel affect m'a rendu insensible au poids épistémique de certaines données alors que je ne dispose d'aucune preuve en faveur de cette idée et que je suis, par conséquent, incapable de la justifier ${ }^{24}$. Cette situation n'a rien d'extraordinaire : ne nous arrive-t-il pas d'entretenir une suspicion à l'égard de certaines de nos croyances dont nous ne parvenons pourtant pas à identifier les faiblesses épistémiques ? Un pessimisme invétéré peut m'inciter de façon parfaitement injustifiée à considérer avec méfiance la croyance que $\mathrm{P}$ du simple fait que je désire par ailleurs que $P$. Parce que je considère ma croyance de premier niveau comme irrationnelle, je peux me sentir dissocié d'un contenu auquel néanmoins je crois. Suis-je, dès lors, victime d'aliénation doxastique ? Le fait que j'appréhende cette croyance comme étant en décalage avec ce que j'estime devoir croire suffit-il ici pour que cette croyance soit aliénée ? La question est, en réalité, de savoir si le critère subjectif doit primer. D'un côté, je me sens extérieur à cette croyance. De l'autre, le critère de sensibilité aux raisons nous empêche de décrire cette situation comme manifestant un cas d'aliénation doxastique au sens où l'entend Moran : ma croyance de premier niveau reflète bien mon appréciation des raisons. Une solution consisterait à considérer cette situation comme un cas intermédiaire : il ne s'agirait ni d'identification ni d'aliénation à proprement parler.

Ensuite, cette interprétation semble certes concorder avec l'idée de Hunter selon laquelle le sentiment d'absence de contrôle qui accompagne les croyances aliénées porte sur l'exercice et/ou le produit de nos facultés cognitives : dans un tel cas, j'échouerais en effet à faire advenir telle croyance épistémiquement désirable et cet échec manifesterait une défaillance de mes capacités délibératives à produire en moi

22 - On peut dire, pour reprendre les termes de Moran (2013,p.124), que dans cette situation la question théorique (Qu'est-ce que je crois ?) est déconnectée de la question délibérative (Que dois-je croire?).

23- Les undercutting defeaters défont la connexion évidentielle qui est censée exister entre telles données et telle croyance. Les rebutting defeaters portent, quant à eux, directement atteinte à la vérité du contenu de la croyance à laquelle la connexion évidentielle est censée aboutir (Pollock 1986) et défont ainsi cette dernière de manière indirecte. Je me base ici sur les analyses d'Owens (2002, p. 390) selon lesquelles juger que tel ensemble de données E est suffisant pour établir la vérité de P implique de considérer que l'ensemble E' supportant non-P ne constitue en réalité aucune raison en faveur de non-P et doit ainsi pouvoir être expliqué de façon compatible avec la vérité de P. En portant atteinte au contenu en faveur duquel elle milite, les rebutting defeaters défont la relation évidentielle de manière indirecte. À ce sujet, voir (Kelly 2014).

24 - Remarquons que le fait qu’une appréciation soit effectivement biaisée n'est pas pertinent, si l'on suit les analyses de Moran. Pour que je mïdentifie à une croyance, il suffit que cette dernière s'ajuste adéquatement à mon appréciation de ses raisons, et ce quelle que soit la qualité épistémique de cette appréciation. En ce sens le fait que je croie faussement que telle croyance est le produit adéquat d'une évaluation objective des raisons, comme dans certains cas de wishful thinking, est parfaitement compatible avec lidentification doxastique. 


\section{Aliénation doxastique, CONTRÔLE ET EXPRESSIVITÉ}

l'état mental conforme au résultat de leur activité. Toutefois, à quel type d'agentivité la sensibilité aux raisons renvoiet-elle ? On peut en effet s'interroger avec Engel (2009) : si l'agentivité doxastique n'est pas censée désigner, dans de tels contextes, les actes cognitifs qui peuvent précéder l'installation d'une croyance et si l'ajustement d'un état doxastique aux raisons ne peut constituer une action, où se situe l'activité à proprement parler ? D’un point de vue phénoménologique, ai-je réellement le sentiment de « contrôler » quoi que ce soit dans ce genre de situation ? Est-ce dès lors d'une absence de contrôle dont je fais l'expérience dans les cas d'aliénation? N'ai-je pas plutôt l'impression qu'un processus qui par principe m'échappe n'opère pas de la manière attendue ?

Enfin, ce compte rendu du vécu d'aliénation doxastique heurte directement TP au sens où il suppose que l'identification doxastique est liée la sensibilité aux raisons épistémiques. Il ne permet donc pas de rendre raison des cas d'aliénations doxastiques non-épistémiques, autrement dit des cas où l'identification requiert précisément l'insensibilité à ce type de raisons ${ }^{25}$. Quand bien même nous accepterions, avec Hieronymi, de concevoir l'identification ainsi entendue comme une forme de contrôle, nous ne trouvons pas d'équivalent non-épistémique à ce mode d'ajustement aux raisons, équivalent à l'aune duquel les cas d'aliénation doxastique non-épistémique pourraient être identifiés par le sujet comme défaillants.

Concluons cette partie. Nous n'avons trouvé aucun sens de la notion de " contrôle » qui nous permette de comprendre le rapport que nous sommes censés entretenir à l'égard de nos états doxastiques aliénés. La phénoménologie de l'aliénation doxastique ne semble donc pas pouvoir être restituée par un sentiment d'absence de contrôle. Elle peut, tout au plus, être décrite comme la perception d'un contraste entre une croyance d'un côté et un état cognitif, affectif et/ou conatif qui la prend pour objet de l'autre. L'agent doxastiquement aliéné « aimerait » que sa croyance s'accorde à ses états de second niveau, peut-être sur le modèle de la manière dont ses états doxastiques s'ajustent ordinairement aux données ou dont ses actions découlent de ses intentions. Prise en son sens littéral, la thèse du contrôle va plus loin : elle fait comme si l'agent aliéné projetait, de manière erronée, la rétro-référentialité de l'intention sur un désir doxastique. Il s'agirait dès lors d'une transposition, psychologiquement assez peu vraisemblable, du concept de contrôle dans un contexte inapproprié plutôt que d'un cas standard à partir duquel une distorsion cognitive pourrait être identifiée. Par conséquent, et contrairement à ce qu'écrit Hunter (2011, p. 228), le contraste, constitutif de l'aliénation doxastique, entre ce qu'il croit et ce qu'il aimerait croire ne paraît pas nécessairement constituer, de ce point de vue, l'indice d'un désordre dans l'exercice des facultés cognitives et intellectuelles de l'agent.

\section{5 - Identification doxastique et expressivité}

L'argument de Moran consiste, comme on l'a vu, à relier étroitement le problème de l'agentivité à celui de l'expressivité. Mais l'expressivité en question est conçue de manière purement épistémique : je suis actif à l'égard de mes croyances lorsque ces dernières expriment adéquatement ma sensibilité aux raisons. Certes, lorsque l'aliénation doxastique est motivée par des raisons épistémiques, le sujet a l'impression que son « vrai Soi » ne s'exprime pas dans la croyance irrationnelle en question et dans la pratique épistémique qu'elle catalyse (Hunter 2011, p. 223-224). Toutefois, le critère d'expressivité qu'il propose est plus large. Rappelons son contenu :

Thèse de l'expressivité : le sujet victime d'aliénation doxastique vit sa croyance comme n'exprimant pas son « moi véritable » ou « profond ».

Cette thèse est, d'un point de vue psychologique, plausible : le sentiment selon lequel ma croyance n'est pas pleinement mienne au sens où elle ne reflète pas ce que je suis, semble bien, la plupart du temps, accompagner le vécu d'aliénation doxastique. Je peux ainsi avoir l'impression que ma personne s'exprime plus authentiquement dans une attitude épistémiquement partiale que dans l'adhésion à un contenu rationnel que je vivrais comme une absence de loyauté à l'égard de mon ami. Dans un tel contexte, une croyance biaisée me paraît bien manifester un aspect substantiel de ma personne en ce qu'elle exprime mon statut d'ami et les engagements que je pense être liés à ce statut.

Toutefois, comment comprendre une telle thèse ? La thèse de l'expressivité affirme que le sujet effectivement victime d'aliénation doxastique vit sa croyance comme n'exprimant pas son « moi véritable ». Cet énoncé peut être compris au moins de deux manières. Soit on considère qu'il suffit que j'appréhende ${ }^{26}$ ma croyance de premier niveau comme étant inexpressive de mon moi véritable, et ce quel que soit la validité objective d'une telle appréhension, pour être victime d'aliénation doxastique. Le vécu d'inexpressivité serait dès lors une condition suffisante de l'aliénation. Soit on considère que, dans les cas d'aliénation doxastique effective, le vécu d'inexpressivité reflète une inexpressivité réelle : le rapport que j'entretiens à ma croyance manifesterait le décalage effectif entre ce que je pense et ce que je devrais penser étant donné mon « moi véritable ». Dans une telle optique, l'inexpressivité réelle serait une composante nécessaire mais non suffisante de l'aliénation doxastique. Cette dernière serait en effet constituée de deux éléments : l'inexpressivité effective de la croyance de premier niveau et, parce qu'il s'agit d'un mode subjectif d'aliénation, l'expérience de cette inexpressivité ou son vécu, qui traduirait ainsi la conscience de ce décalage. Cette composition double ne saurait cependant préjuger 


\section{Aliénation doxastique, CONTRÔLE ET EXPRESSIVITÉ}

de la nature du rapport entre ces deux éléments. Il se pourrait ainsi, par exemple, que le second accompagne toujours empiriquement le premier ${ }^{27}$. Inversement, pour les cas, s'ils existent, où nous prenons conscience de la fausseté de tel sentiment passé d'inexpressivité, une telle approche consisterait à dire que nous ne cessons pas d'être victimes d'aliénation doxastique mais que nous découvrons qu'en réalité nous ne l'étions pas.

Nous partirons de la seconde interprétation de cette thèse car elle nous semble plus proche de certaines intuitions de Hunter. Si le rapport subjectif que le sujet entretient à sa croyance est un aspect central de l'aliénation doxastique, l'aliénation ne se réduit pas à ce rapport subjectif car le vécu en question est censé indiquer un dysfonctionnement plus général - même si ce dysfonctionnement est difficile à décrire - dans l'exercice de nos facultés. La première conception fait de l'aliénation une réalité dépendant uniquement du vécu ponctuel de l'individu : ce phénomène varierait au gré des aléas de ma vie mentale et serait ainsi tributaire des méta-attitudes et des sentiments de dissociation plus ou moins contingents, évanescents et superficiels qui peuvent surgir en son sein ${ }^{28}$. En partant de l'idée selon laquelle l'aliénation doxastique désigne au contraire une scission importante au sein de notre vie mentale, j'interroge la valeur épistémique du vécu d'inexpressivité doxastique. Deux séries de questions structureront alors respectivement nos deux prochaines sections. D'une part, quels sont les critères d'identification de l'expressivité d'une croyance ? Autrement dit, à supposer que les cas d'aliénation échouent à exprimer le Soi, où situer l'instance expressive du Soi à partir de laquelle le sujet aliéné puisse identifier cet échec ? D’autre part, ce vécu est-il nécessairement fiable? N'est-il pas possible d'imaginer des situations au cours desquelles des croyances expressives du Soi occasionnent pourtant une impression de distance à soi ? En d'autres termes, la phénoménologie de l'aliénation est-elle immunisée face à l'erreur?

\subsection{Hiérarchie d'attitudes et « Problème d'Au- torité »}

Indépendamment de toute considération phénoménologique, le thème de l'expressivité paraît fécond pour caractériser l'aliénation : il semble bien que quelque chose du sujet 27-En ce sens, dans les cas d'aliénation authentique, le rapport subjectifen question serait nécessairement factif. Remarquons que, formulée de manière générale, la seconde possibilité nïmplique pas que des cas dinexpressivité réelle non-appréhendée comme telle et/ou des cas d'appréhension erronée d'inexpressivité existent. Nous pourrions considérer qu'une conscience immédiate capte toujours cette inexpressivité ou que le vécu dïnexpressivité, dès lors qüll advient, est nécessairement fiable. En revanche, selon cette seconde possibilité, les cas d'inexpressivité réelle non captés par ce type de vécus, sïls existent, ne sauraient être à proprement parler des cas d’aliénation doxastique puisqü̈l leur manquerait cet écho subjectif. Inversement, sül existe des situations au cours desquelles le sujet vit telle croyance comme n'exprimant pas son moi profond en dépit du fait qu'elle l'exprime en réalité, alors cette même croyance ne pourrait être considérée comme aliénée. Mais, comme me le fait remarquer un évaluateur anonyme, ce cas de figure ne constitue pas nécessairement un problème pour la thèse de l'expressivité prise en son sens le plus général. La sous-section 5.2 examinera ces deux derniers cas de figure.

28 - En optant pour cette seconde conception, nous choisissons en un sens de renouer avec lidée, présente au sein d'une certaine tradition philosophique, selon laquelle le concept d'aliénation caractérise une forme essentielle de dissociation de soi ou de scission interne à lïndividu aliéné, et plus précisément ici à sa vie mentale. On pourrait cependant répondre que la simple émergence d’une méta-attitude négative suffit à exprimer un dysfonctionnement plus large. Nest-ce pas cependant surestimer la portée révélatrice de nos états mentaux? N'est-il pas possible de concevoir que telle appréhension d’inexpressivité doxastique dépend, par exemple, d’une simple humeur passagère et n'exprime ainsi rien de substantiel sur notre vie mentale?

29-Dans l'exemple de Hunter, linsensibilité, c'est-à-dire le rapport sélectif aux données, ne doit pas nécessairement caractériser la réception des données mais, essentiellement, la formation de la croyance qui en résulte. La mère considère que, quelle que soit son évaluation des données, elle ne doit pas croire que P. En un sens, elle ferait d'autant plus preuve de loyauté que son approche des données n'est pas biaisée.

30 - Bien quïl existe des valeurs épistémiques, nous nous focaliserons ici sur les valeurs non épistémiques, et plus précisément sur les valeurs morales.

31 - J'emprunte cette expression à Elster (2010, p.100). 


\section{Aliénation doxastique, CONTRÔLE ET EXPRESSIVITÉ}

Si le « toxicomane malgré lui » est inapte à traduire ses valeurs en actes (Watson 2008, p. 170), la mère ferait, quant à elle, l'expérience de son incapacité à faire en sorte que sa croyance reflète sa valeur. En ce sens, une partie plus substantielle de sa personne se manifesterait dans cette attitude de second niveau que dans le fait d'ajuster ses croyances de premier niveau aux données disponibles : son attitude de second niveau exprimerait dès lors, et serait perçue par elle comme exprimant ce qu'elle devrait penser si son comportement doxastique était en harmonie avec ses valeurs. On ne peut donc pas dire, sans plus, que la méta-attitude exprime dans cet exemple ce qu'elle est puisqu'elle vit précisément mal le fait de ne pas être, du moins sur le moment, le type de mère qu'elle aspire à être. Quand bien même son comportement actuel ne serait pas en adéquation avec ce que lui dicte cette valeur, cette adhésion axiologique peut néanmoins être conçue comme un aspect essentiel de sa personne. Sa métaattitude serait ainsi comme un signal du contraste entre sa croyance et ce qu'elle devrait croire si son comportement doxastique était ajusté à ses aspirations véritables. En ce sens, et pour reprendre les mots de Hunter, elle considérerait que cette méta-attitude, contrairement à sa croyance de premier niveau, exprime son « moi profond ».

Une telle conception de l'accès à cette partie substantielle de soi, à l'aune de laquelle un état mental pourra être dit « aliéné », est-elle correcte ? L'explicitation de l'aliénation en termes d'attitudes mentales hiérarchisées semble problématique à bien des égards. N'est-il pas possible, tout d'abord, d'entretenir des attitudes de second ordre elles-mêmes aliénées ? Je peux très bien considérer une attitude de second niveau comme n'étant pas pleinement mienne et ressentir dès lors cette évaluation négative de ma croyance de premier niveau comme extérieure à moi. Je suis, par exemple, susceptible d'avoir honte du sentiment de désapprobation que m'inspire une croyance de premier niveau et ainsi de ne pas m'identifier à cette méta-attitude de désapprobation. On pourrait toutefois répondre à cette première objection que le fait qu'une attitude puisse en principe être supplantée par une attitude de niveau supérieur ne saurait signifier qu'elle l'est nécessairement. $\mathrm{Si}$, dans notre exemple, la mère entretient simultanément - et uniquement - cette croyance combinée à la croyance selon laquelle ne devrait pas l'entretenir, pourquoi ne devrions-nous pas considérer cette hiérarchie d'attitudes comme suffisamment expressive de sa personne? Le fait qu'une évaluation négative de niveau supérieur puisse voir le jour doit-il être conçu comme un argument pertinent contre la valeur expressive de sa méta-attitude actuelle?

Cette réponse nous semble cependant manquer sa cible en ce qu'elle ne saisit pas la portée de l'objection initiale. Cette dernière consiste, en réalité, à appliquer à la question des croyances ce que Hinshelwood (2013) nomme le «problème d'autorité » lié, selon lui, à toute explicitation en termes hiérarchiques des concepts d'identification et d'aliénation. Ce problème, initialement formulé par Watson $(2008)^{32}$, peut être résumé ainsi : pourquoi le niveau hiérarchique au sein d'une échelle d'attitudes conférerait-il à tel état mental une autorité spéciale en matière d'expressivité ? Il ne s'agit pas de remettre en cause le fait qu'une méta-attitude puisse être aliénée en invoquant une attitude de niveau supérieur potentiellement négative à son encontre mais de questionner les fondements de la présomption d'identification en faveur de cette même méta-attitude. Le modèle hiérarchique ne peut en effet se contenter de l'absence de méta-attitude négative : il implique au contraire que l'identification elle-même soit actuellement attestée par une méta-attitude positive. Dit autrement, pour que l'agent s'identifie à sa croyance selon laquelle il ne devrait pas enfreindre, dans son comportement doxastique, certains devoirs, ne faut-il pas que cette croyance, et donc l'évaluation qu'elle enveloppe, fasse elle-même l'objet d'une évaluation positive de niveau supérieur ? On voit cependant que cette quête de l'item psychique non-aliénant pose un problème de régression à l'infini : comment une attitude quelconque pourrait-elle, par conséquent, avoir autorité pour exprimer pleinement le Soi ? Autrement dit, à l'aune de quelle instance mentale pourrait-on décrire tel état comme aliéné ? Le problème d'autorité serait inhérent à toute tentative d'explicitation hiérarchique du concept d'identification.

Il semble, tout d'abord, possible de répondre à cette objection sans remettre en cause le modèle hiérarchique. Revenons à la thèse selon laquelle seule l'aliénation devrait être expliquée. Frankfurt (1999, p. 103-107) lui-même a proposé l'idée selon laquelle la notion de « satisfaction » pouvait expliquer l'identification aux méta-attitudes de façon à éviter toute possibilité de régression à l'infini. La satisfaction est explicitée, de manière négative, par l'absence d'item psychique antagoniste : un agent est « satisfait » de sa condition lorsqu'il ne ressent aucune résistance à celle-ci ni de tendance ou d'inclination à l'altérer (Frankfurt 1999, p. 103-104). Le fait d'être satisfait d'un état mental ne requiert dès lors aucunement d'entretenir une attitude cognitive, conative ou affective quelconque à son égard (Frankfurt 1999, p. 104). On peut, dès lors, appliquer aux méta-attitudes l'idée de satisfaction. Un agent s’identifie à son désir de ne pas se droguer s'il est satisfait de sa volition de niveau supérieur de ne pas désirer se droguer. En appliquant cette solution aux phénomènes doxastiques, on pourrait dire que je m'identifie à la méta-attitude qui prend ma croyance de premier niveau pour objet si je suis satisfait de cette même attitude. Dès lors, c'est parce que je suis satisfait de cette évaluation négative que je vis ma croyance de premier niveau comme aliénée. Nous n'aurions plus besoin d'attitude de niveau supérieur qui garantirait l'identification à la méta-attitude génératrice d'aliénation. On peut alors parler d'une présomption défaisable d'identification à l'égard de ces méta-attitudes : en l'absence de défaiseurs, nulle attitude de niveau supérieur ne serait nécessaire afin que nous considérions comme nôtre la méta-attitude initiale. Une asymétrie existerait donc entre la satisfaction et l'identification à pro- 


\section{Aliénation doxastique, CONTRÔLE ET EXPRESSIVITÉ}

prement parler. Afin de m'identifier ou de me sentir extérieur à une croyance de premier niveau, je devrais ultimement être satisfait de la méta-attitude évaluative qui la prend pour objet.

Une telle explicitation est censée éviter le problème d'autorité. Toutefois, une question vient assez naturellement à l'esprit : pourquoi ne pas appliquer le concept de satisfaction dès le premier niveau, ce qui éviterait de postuler une différence, difficilement justifiable, entre les critères d'identification selon la place hiérarchique des attitudes concernées (Hinshelwood 2013, p. 251) ? Le niveau d'une attitude ne semble pas, en effet, affecter sa nature : comment comprendre qu'à partir d'un niveau $\mathrm{X}$ la satisfaction devienne un critère légitime d'identification ? Pourquoi, autrement dit, ne pas considérer que la présomption défaisable d'identification concerne directement nos croyances de premier ordre et, plus généralement, toute attitude mentale dès lors qu'aucune méta-attitude négative ne surgit? Une présomption par défaut d'identification caractériserait ainsi nos états mentaux de premier niveau en l'absence d'évaluation négative. Le modèle hiérarchique ne serait dès lors pertinent que pour les cas d'aliénation qui, seuls, auraient à être expliqués.

Cette thèse est attractive. Elle semble toutefois restrictive si on l'applique à l'idée d'expressivité. Comme l'a souligné Bratman (1996, p. 7), l'absence de méta-attitude négative peut être liée à des causes contingentes et n'avoir aucune vertu expressive ou révélatrice concernant l'individu qui entretient l'état mental de premier niveau. L'absence d'un tel jugement de niveau supérieur ne peut à elle seule garantir l'identification à l'attitude mentale de premier niveau. Imaginons que, parce que son esprit est occupé à d'autres considérations, la mère n'ait actuellement aucune méta-attitude à l'encontre de sa croyance de premier ordre. Il n'est toutefois pas absurde, si l'on considère l'ensemble de sa vie mentale et si l'on songe à la manière dont elle évalue ordinairement ses réactions et hiérarchise ses motifs d'actions, d'affirmer qu'elle devrait ou aurait dû, pour des raisons de cohérence, entretenir une méta-attitude négative à l'égard de sa croyance de premier niveau. Si l'on conçoit son identité en un sens diachronique, il paraît important, du point de vue du critère même d'expressivité, de pouvoir relier ses méta-attitudes au reste de sa vie mentale. La simple absence de fait d'un jugement de second ordre peut-elle dès lors valoir comme satisfaction ? Si le concept de satisfaction se réduit à l'absence ponctuelle de tendance antagoniste, la réponse est positive. Toutefois, ce concept ne perdrait-il pas alors une partie de sa plausibilité ? Le fait de ne pas juger, à tel instant $\mathrm{T}$, qu'un état doxastique ne reflète pas, par exemple, telle valeur à laquelle j'adhère profondément signifie-t-il nécessairement qu'il ne viole effectivement pas cette même valeur?

\subsection{Le problème des méta-attitudes inexpres- sives}

Interrogeons à présent le contenu des méta-attitudes. Si le sujet doxastiquement aliéné perçoit sa croyance comme inexpressive d'une partie substantielle de sa personne, cette appréhension immédiate ne peut-elle pas être erronée ? En d'autres termes, le contenu du jugement de second niveau ne peut-il pas lui-même être en décalage avec, par exemple, les valeurs les plus fortement enracinées chez le sujet, que ces dernières soient ou non l'objet de méta-attitudes?

La mère, écrit Hunter (2011, p. 225), peut se sentir temporairement étrangère à sa croyance épistémiquement rationnelle selon laquelle son fils se drogue parce qu'elle estime que cette croyance n'est pas en harmonie avec ce qu'exige son amour maternel. Suite à l'examen des différents problèmes liés au modèle hiérarchique, nous avons mentionné l'importance, du point de vue du critère même d'expressivité, de pouvoir relier ses méta-attitudes au reste de sa vie mentale. Faute de pouvoir élaborer une conception diachronique générale de l'expressivité, partons de cette intuition. Imaginons alors qu'après une certaine réflexion, la mère en vienne à prendre conscience du fait que, tout bien considéré, c'est en assumant sa culpabilité, ainsi qu'en agissant sur la base de cette assomption, qu'elle exprime véritablement son amour pour lui et qu'elle fait réellement preuve de loyauté à son égard. Admettons, pour les besoins de la cause, que cette prise de conscience ne signifie pas qu'elle révise sa croyance initiale concernant ses devoirs de mère, comme si elle découvrait par exemple que la reconnaissance de la culpabilité de son fils est en réalité, et contrairement à ce qu'elle croyait, plus conforme à ce que doit être l'amour maternel. Imaginons plutôt qu'elle considère à présent, et avec raison, que la reconnaissance de la culpabilité de son fils découle de sa conception effective de l'amour maternel, autrement dit de ce qu'elle pense et a toujours, en réalité, pensé être ses devoirs de mère.

Les motifs de révision de cette méta-attitude concernant ce qu'elle doit croire peuvent avoir différentes sources. Elle peut réaliser que son méta-jugement n'était pas adéquat à sa conception effective grâce à une réflexion portant explicitement sur le contenu de sa croyance concernant la loyauté maternelle et ses implications, réflexion qui consisterait, entre autres, à tester cette conception en la confrontant à ce cas de figure inédit (à savoir le fait d'être en présence de données qui l'incitent à croire ce genre de choses sur son fils) ou à d'autres cas similaires. On pourrait également imaginer qu'elle révise son méta-jugement en orientant son regard plus étroitement sur elle-même : elle comprend, après avoir passé en revue ses actions et réactions passées et tenté d'identifier ses dispositions profondes, que l'assomption de la culpabilité de son fils s'avère en réalité bien plus cohérente avec ces dernières. Elle prend ainsi conscience du fait, d'une part, que son comportement était tacitement gouverné par la maxime selon laquelle l'amour maternel implique un devoir de sincérité et, d'autre 


\section{Aliénation doxastique, CONTRÔLE ET EXPRESSIVITÉ}

part, qu'elle adhère explicitement à cette maxime quand elle évalue réflexivement son contenu.

Greco (2014) a examiné la possibilité de situations akratiques au cours desquelles un sujet croit que $\mathrm{P}$ et croit faussement qu'il doit croire que non- $\mathrm{P}^{33}$. Remarquons qu'un individu en proie à un sentiment d'aliénation doxastique pour des motifs axiologiques peut se trouver dans une situation analogue. La méta-attitude négative initiale, à savoir ici ce que l'agent juge spontanément ne pas devoir croire pour des raisons axiologiques, peut en effet ne pas être conforme au contenu réel de ses adhésions. Sur la base des considérations précédemment exposées, la mère serait ainsi amenée à réviser non pas ses valeurs (puisqu'elles sont censées rester intactes) mais sa méta-attitude antérieure et, par conséquent, le sentiment de distance à soi que cette méta-attitude a catalysé. Elle peut dès lors s'identifier rétrospectivement à cette croyance initialement appréhendée comme étrangère. Les raisons de cette réévaluation ne sont pas "externes ", au sens où Williams (1979) entend ce terme, puisque les valeurs sur lesquelles elles se fondent figurent bien, quoi qu'elles n'aient peut-être jamais été thématisées, parmi les raisons auxquelles l'individu peut avoir accès s'il délibère, et plus précisément s'il explicite le contenu de certaines de ses adhésions axiologiques.

Quelle que soit la position hiérarchique de l'attitude censée les exprimer, les valeurs ne sont pas nécessairement transparentes au sujet qui y adhère. Si toutefois les valeurs peuvent, au moins en partie, être conçues comme un certain type de croyances, à savoir des croyances axiologiques, comment comprendre que je puisse faire erreur sur ce que je crois surtout si, comme dans le cas des valeurs fortement enracinées, ce que je crois est censé être constitutif de ce que je suis ? Comment, en effet, expliquer le décalage possible entre ce qu'on croit devoir croire pour des raisons axiologiques et ce qu'on doit effectivement croire pour ces mêmes raisons ${ }^{34}$ ? Reprenons notre exemple. Ce qu'échoue à saisir sa méta-attitude semble être au moins une partie du contenu de son adhésion axiologique et, plus précisément, de ses implications doxastiques. Elle a toujours tacitement considéré qu'une attitude réaliste était plus conforme à son devoir mais elle a eu un moment de faiblesse en considérant qu'elle ne devait pas affronter la réalité, dans ces circonstances, en n'ajustant pas sa croyance aux données. Comment expliquer, toutefois, l'opacité possible des croyances axiologiques?
La conception dispositionnaliste des croyances que propose Schwitzgebel (2002) permet de saisir une telle possibilité. Selon Schwitzgebel, chaque croyance est liée à un " stéréotype dispositionnel ", c'est-à-dire à un ensemble de dispositions que nous associons conceptuellement à sa possession. Les dispositions génériques associées aux croyances sont au nombre de trois : une croyance se caractérise par des dispositions comportementales (que ce comportement soit verbal ou non), cognitives (la disposition à effectuer un certain nombre d'inférences à partir du contenu de la croyance en question) et phénoménales, à savoir la disposition à entretenir un certain nombre d'expériences conscientes (Schwitzgebel 2002, p. 252). Concentrons-nous sur les dernières : toutes choses égales par ailleurs, croire que P c'est être disposé, par exemple, à ressentir de la surprise si non-P mais également, ce qui retiendra notre attention, à juger que $\mathrm{P}$, c'est-à-dire à entretenir consciemment la pensée que P. Dire qu'un jugement manifeste habituellement une croyance ne saurait toutefois signifier que les jugements ne peuvent, par principe, être en décalage avec nos croyances (Schwitzgebel 2010) : je peux sincèrement juger que le sexisme est moralement inacceptable et entretenir des attitudes (comportementales, phénoménales et cognitives) massivement sexistes, attitudes qui peuvent parfaitement ne susciter en moi aucun retour critique. J'ignore, en ce cas, avoir des croyances sexistes.

L'idée qui nous importe est ici la suivante : la conscience de nos jugements peut biaiser nos auto-attributions de croyances. Selon Schwitzgebel, je peux croire que je crois que le sexisme est moralement inacceptable sur la base de mes jugements portant sur la vérité de ce contenu propositionnel (lorsque, par exemple, je suis confronté à un argumentaire sexiste) alors que mon comportement et mes réactions affectives expriment globalement la croyance contraire. On peut donc, du fait du jugement que $\mathrm{P}$, croire faussement qu'on croit que $\mathrm{P}^{35}$. Du fait de son jugement de son second niveau selon lequel son statut de mère devrait lui interdire ce type de pensée, la mère croit que sa croyance va à l'encontre de sa valeur et de ses implications doxastiques alors que ce même jugement biaise en réalité son accès au contenu de sa valeur. Il y a donc bien, ici, un décalage entre une croyance axiologique et un jugement qui prétend pourtant restituer son contenu. C'est pourquoi une méta-attitude évaluative ne correspond pas nécessairement aux valeurs profondes des agents, et ce quel que soit le rapport que la méta-attitude prétend entrete-

33 - Le cas de figure qui intéresse Greco dans cet article diffère de la situation décrite en 4.2 (p. 16) du point de vue du contenu de la croyance de second niveau. Il faut ainsi distinguer le fait, analysé par Greco, de croire faussement, mais de manière épistémiquement justifiée, que l'on doit croire que non-P du fait de croire (que cette croyance soit vraie ou fausse) irrationnellement que l'on ne doit pas croire que P, ce qui correspond au cas de figure que nous envisagions. Ces deux situations doivent, à leur tour, être distinguées du cas de figure où un individu entretient un sentiment erroné d'aliénation doxastique axiologiquement motivée. Dans ce dernier cas, il croit (que cette croyance soit épistémiquement justifiée ou non) quill ne doit pas croire que P pour des motifs axiologiques. Le sens de « doit » diffère en ce qu’il n'est pas épistémique : il s'agit pour lui de former non pas des croyances vraies mais des croyances conformes à ce quỉl pense être ses valeurs. Il faut dès lors, en tout rigueur, distinguer le type de raisons pour lesquelles on doit ou on pense devoir entretenir ou non telle croyance, d'une part, des raisons pour lesquelles on croit qu'on doit ou non entretenir telle croyance pour ce type de raisons, d'autre part.

34 - Le sujet ne fait donc pas une erreur portant sur l'existence d'un jugement de second niveau (la méta-attitude affirmant que sa croyance contredit telle adhésion axiologique) mais, au contraire, sur la validité de son contenu, c'est-à-dire de la question de savoir si telle est réellement son adhésion axiologique (par exemple, sa conception des devoirs liés au statut de mère, d'ami, etc.) et donc sïl y a bien contradiction entre son attitude de premier niveau et sa valeur. Si les jugements peuvent être décrits comme des réalités mentales occurrentes et conscientes, il semble en effet difficile, hormis peut-être dans certains cas pathologiques, de considérer qu'on puisse se tromper sur l'avènement d’un jugement en soi. 35-Remarquons tout de même qu'un tel décalage est plus paradoxal qu’iln'y paraît sil'on songe au fait que parmi les manifestations de la croyance que P figure également l'autoattribution de la croyance que P. Sur cette question, voir (Schwitzgebel 2011). 


\section{Aliénation doxastique, CONTRÔLE ET EXPRESSIVITÉ}

nir à l'égard de leur contenu ${ }^{36}$.

Nous avons vu que certaines émotions, comme la honte, le sentiment de désapprobation ou l'indignation, accompagnaient souvent les attitudes aliénées. Or, le même type d'analyse peut être reproduit eu égard aux émotions. De même qu'un jugement de second niveau ne reflète pas nécessairement nos croyances axiologiques authentiques, une émotion portant sur l'une de nos attitudes mentales peut parfaitement échouer à exprimer notre rapport effectif à telle valeur. Intuitivement, il semble pourtant que les émotions constituent des révélateurs fiables de nos adhésions. Le fait de ressentir de la surprise, pour prendre un exemple paradigmatique (Davidson 2002, p. 73), signifie bien que j'ai conscience du contraste entre mon ancienne croyance et la nouvelle information que j'acquiers. En ce sens, la surprise peut me révéler certaines de mes attentes dont je n'avais pas conscience. Certaines émotions, comme l'indignation, qui enveloppent des croyances axiologiques, paraissent quant à elles constituer des indices du fait qu'une de nos valeurs a été violée par le monde (Livet 2002) : si je découvre que l'esclavage existe encore dans certaines contrées, mon indignation peut être comprise comme une réaction émotionnelle à la violation que le monde inflige à ma valeur. On peut dès lors considérer qu'une telle émotion est susceptible de me révéler, dans certains cas, les valeurs auxquelles j'adhère. Cette même vertu révélatrice semble pouvoir être attribuée aux réactions émotionnelles que suscitent en nous non seulement le monde mais nos propres attitudes mentales. Si la mère ressent de la honte du fait qu'elle croit que $\mathrm{P}$, c'est bien, à première vue, que cette croyance viole une norme à laquelle elle adhère. La croyance en la culpabilité de son fils n'exprimerait donc pas adéquatement sa valeur, ce qu'est censée exprimer l'émotion en question. Toutefois, nos émotions constituent-elles nécessairement un mode d'accès direct et fiable à nos adhésions axiologiques?

Comme on a pu le montrer, nos émotions peuvent certes constituer des indices de ces adhésions axiologiques mais cela ne saurait signifier qu'elles n'ont pas de " conditions d'adéquation » (Tappolet 2000) et, par conséquent, qu'elles ne sont pas ouvertes à révision (Livet 2002). Ainsi, une émotion d'admiration est adéquate ou appropriée si la personne que j'admire est réellement admirable. L'évaluation des émotions est ainsi faite, dans ce contexte, en fonction de leur adéquation ou correspondance à des faits axiologiques (Tappolet 2000, p. 170-175). Sans entrer dans le débat portant sur l'existence de tels faits, constatons que ce type d'idée peut être appliqué au rapport émotionnel que nous entretenons à l'égard de nos propres attitudes, en l'occurrence ici à l'égard d'une croyance de premier niveau, et ce du point de vue de leur prétention à révéler nos adhésions axiologiques effectives.

Imaginons, ainsi, que la mère ressente de l'indignation à l'égard du fait que sa croyance de premier niveau concernant son fils s'ajuste purement et simplement aux données. On peut, à première vue, concevoir ce sentiment d'indignation face à cette croyance comme l'indice d'une non-conformité de cet état doxastique à sa croyance concernant ses devoirs maternels. Or, une réflexion du type de celle que nous avons envisagée plus haut pourrait lui apprendre que sa conception de l'amour maternel n'a pas, en réalité, cette implication. Elle peut ainsi lui fournir une raison de ne plus entretenir cette émotion ainsi que de ne plus ressentir cette croyance comme étrangère à elle. Autrement dit, cette réflexion est susceptible de lui révéler que sa réaction initiale à sa croyance constituait une « réponse émotionnelle inappropriée » (Mulligan 1998, p. 161) non à l'égard de certains traits du monde mais à l'égard de l'attitude doxastique qu'elle avait pour objet. L'évaluation de l'adéquation de cette émotion ne serait pas faite, dans ce contexte, selon sa conformité à des faits axiologiques indépendants de son système évaluatif, puisque nous n'entrons pas dans le débat portant sur l'existence de tels faits, mais à l'aune de ses adhésions axiologiques effectives. La mère prendrait ainsi conscience de l'expressivité authentique de son état doxastique antérieur et de l'inadéquation axiologique de l'émotion qu'il a suscitée. Si cette analyse est correcte, alors le vécu d'inexpressivité et l'émotion qui l'accompagne ont également des conditions d'adéquation. Imaginons que malgré cette prise de conscience, l'émotion initiale persiste : cette indignation vécue ne sera plus un indicateur d'aliénation mais constituera elle-même un état mental aliéné. Qu'elle subsiste ou non, l'émotion initiale a été corrigée du point de vue de sa " prétention » à révéler les valeurs de l'individu qui l'entretient. Elle ne justifie plus une auto-attribution de croyances axiologiques et constituait donc bien une source défaisable d'identification de valeurs ${ }^{37}$.

Si un certain type de réflexion est susceptible de me révéler mes adhésions authentiques, on voit que le critère d'expressivité, tel que nous l'entendons, n'implique pas que l'on se contente d'enregistrer l'écho subjectif immédiat que suscitent en nous nos attitudes de premier niveau. Il porte au contraire, selon notre interprétation, sur l'adéquation des croyances initiales aux adhésions axiologiques réelles de l'individu qui les entretient. La phénoménologie de l'aliénation doxastique ne saurait dès lors être décisive : nous ne sommes pas a priori immunisés contre l'erreur d'identification en matière d'aliénation. Le vécu d'inexpressivité ne garantit pas l'inexpressivité effective de l'état qu'il prend pour objet et sa présence ne suffit donc pas, conformément à notre définition, à en faire

36 - La possibilité de s'auto-attribuer de manière erronée telle croyance peut être appliquée aux attitudes de premier niveau. Nous sommes partis du principe d’une transparence à soi concernant ces dermières mais il est possible de croire faussement qu'on croit que P et de ressentir cette même croyance comme aliénée alors que nous ne faisons en réalité que juger que P sans que ce jugement ne reflète un profil dispositionnel plus large auquel correspondrait la croyance que $P$.

37-En ce sens, les émotions sont seulement des justifications de première apparence (Livet 2002, p. 168) pour nos auto-attributions de croyances axiologiques. Peut-on, dès lors, inférer de nos émotions nos adhésions axiologiques authentiques? Selon Livet, lïmagination des émotions conséquentes à la réalisation ou à la non-réalisation de telle valeur constitue un test nous permettant de détecter nos valeurs effectives (Livet 2002, p. 182-188). 


\section{Aliénation doxastique, CONTRÔLE ET EXPRESSIVITÉ}

un état authentiquement aliéné. Dans les cas d'identification doxastique rétrospective, c'est bien ce sentiment erroné de distance à soi qui est inadéquat. La situation inverse peut également advenir : je peux comprendre que j'aurais dû ressentir comme étrangère à moi telle croyance antérieure, par exemple parce qu'elle allait à l'encontre de mes options axiologiques fondamentales et échouait, dès lors, à exprimer un aspect constitutif de ma personne. En l'absence d'inexpressivité vécue, cette croyance passée ne saurait, également, être décrite comme aliénée. Si l'inexpressivité réelle et son écho subjectif sont les composantes de l'aliénation doxastique ainsi considérée, nous avons alors affaire au même type de situations intermédiaires que celles analysées précédemment (cf. 4.2) : ni l'identification ni l'aliénation ne peuvent adéquatement caractériser les cas où un décalage existe entre l'expressivité réelle et l'expressivité vécue d'un état.

L'aliénation subjective est, comme nous l'avons exposé en introduction, censée épouser le point de vue de l'agent, et donc par exemple ses valeurs. Un tel concept n'implique cependant pas que ce point de vue soit immédiatement accessible à l'individu lui-même ni que ses réactions immédiates le reflètent invariablement. L'aliénation subjective ainsi entendue ne suppose dès lors pas que nous soyons immédiatement conscients de nos valeurs et que nos vécus d'inexpressivité soient infaillibles. Notre interprétation de la thèse de l'expressivité reste dans le cadre d'une analyse de l'aliénation subjective bien qu'elle ne fasse pas de l'écho subjectif suscité par un état mental une condition suffisante de l'aliénation de ce même état. Il ne s'agit en effet pas ici d'aliénation « objective ", si l'on comprend cette dernière comme résultant d'une imputation potentiellement indépendante de tout rapport que le sujet peut entretenir à ses états mentaux : d'une part, parce que le concept d'aliénation mobilisé désigne un état qui inclut nécessairement une composante vécue ; d'autre part, car même si notre perspective nous incite à récuser dans certains cas le rapport subjectif que le sujet entretient à ses attitudes, c'est au nom d'un point de vue qu'il peut avoir sur celles-ci s'il explore le contenu de ses adhésions axiologiques actuelles. L'aliénation doxastique est dès lors toujours au moins dépendante du point de vue qu'un sujet a potentiellement sur lui-même. Tout le problème, si l'on ne désire pas plaquer sur les agents des normes qui ne sont pas les leurs, réside toutefois dans la manière de théoriser les conditions subjectives d'identification de ces mêmes valeurs ${ }^{38}$.

\section{6 - Conclusion}

Nous pouvons nous sentir, pour diverses raisons, étrangers à l'égard de certaines de nos croyances du fait de la pluralité des standards à l'aune desquels nous pouvons les évaluer et de la diversité des méta-attitudes qu'elles sont susceptibles d'occa- sionner. En ce sens, la notion d'aliénation doxastique renvoie bien, d'une part, à une réalité vécue et ne préjuge pas, d'autre part, de la rationalité des croyances en question. Le problème est alors de comprendre en quoi elle consiste. Nous avons montré que la tentative de décrire, d'un point de vue psychologique, l'aliénation comme une absence de contrôle était problématique. La voie expressiviste semble plus prometteuse en ce qu'elle paraît éclairer, de façon plausible, le sentiment d'extériorité que l'on entretient à l'égard des croyances aliénées. Mais elle suppose, afin d'être poursuivie, de trouver une manière de rendre compte de l'aliénation doxastique qui évite le problème d'autorité et celui des méta-attitudes inexpressives. Nous n'adhérons pas, en effet, nécessairement aux standards à l'aune desquels nous évaluons immédiatement nos états doxastiques. En ce sens, une attitude peut, quelle que soit sa situation hiérarchique, échouer à exprimer adéquatement tel aspect constitutif de notre personne, comme nous l'avons illustré à travers le cas des valeurs. Il s'agit dès lors de retravailler le concept d'expressivité et de formuler des conditions claires d'attestation de l'expressivité des croyances afin de développer une conception de l'aliénation doxastique subjective qui intègre cette possibilité d'échec.

\section{Remerciements}

Je remercie les Archives Henri Poincaré ainsi que le Comité de la Société de Philosophie Analytique de m'avoir permis de participer au congrès de la SoPhA (Montréal, 2015) au cours duquel j'ai pu présenter la plupart des idées émises dans cet article.

38 - Comment identifier nos adhésions axiologiques ? Doit-on, par exemple, recourir à des critères contrefactuels d’identification comme nous l'avons suggéré dans cette section? Frankfurt (1988a, p. 165) remarque, à ce propos, quill peut être difficile de répondre à la question de savoir, par exemple, ce que désire " réellement » une personne. Cette dernière peut en effet entretenir des attitudes profondément ambivalentes à l'égard d'un même objet, avoir des désirs irréductiblement conflictuels, etc. Dans ce type de cas, l'ïncohérence est plus profonde : le sujet n'est pas aliéné par un désir en conflit avec ses volitions de second niveau mais bien divisé en ce qu’une scission affecte son système de volitions lui-même. 
REVUE

DE LA SOCIÉTÉ

DE PHILOSOPHIE

DES SCIENCES

\section{RÉFÉRENCES}

ANSCOMBE, Elizabeth. 2002. Lïntention. Paris: Gallimard. BENNETT, Jonathan.1990. Why is Belief Involuntary? Analysis, 50(2), 87-107. Article

BRATMAN, Michael. 1996. Identification, Decision, and Treating as a Reason. Philosophical Topics, 24(2), 1-18. Article

BUCKAREFF, Andrei. A. 2008. Action and Doxastic Control. The Asymmetry Thesis Revisited. European Journal of Analytic Philosophy, 4(1), 5-12.

COHEN, Jonathan. 1989. An Essay on Belief and Acceptance. Oxford: Oxford University Press. Livre

DAVIDSON, Donald. 1991. Paradoxes de lïrrationalité. Nîmes: Éditions de l'Éclat. Livre

DE BRABANTER, Philippe, NICOLAS, David, STOJANOVIC, Isidora, VILLANUEVA, Neftali. 2007. Les usages déférentiels. In BOUVIER Alban et CONEIN Bernard (dir.). L'épistémologie sociale. Une théorie sociale de la connaissance, Paris: Éditions de l'EHESS. 139-162.

ELSTER, Jon. 1986. Le laboureur et ses enfants : deux essais sur les limites de la rationalité. Paris: Éditions de Minuit.

ELSTER, Jon. 1990. Psychologie politique, Veyne, Zinoviev, Tocqueville. Paris: Éditions de Minuit.

ELSTER, Jon. 2003. Proverbes, maximes et émotions. Paris: PUF. Livre

ELSTER, Jon. 2010. Lïrrationalité. Traité critique de l'homme économique. Paris: Éditions du Seuil.

ENGEL, Pascal. 2007. Akrasia pratique et akrasia épistémique. Le philosophoire, 29(2), 63-79. Article

ENGEL, Pascal. 2009. Epistemic responsibility without epistemic agency. Philosophical Explorations, 12(2), 205-219. Article

FOLEY, Richard. 1994. Pragmatic Reasons for Belief. In JORDAN Jeff (dir.). Gambling On God, Essays on Pascal's Wager. Lanham, Md: Rowman \& Littlefield. 31-46.

FRANKFURT, Harry G. 1971. Freedom of the Will and the Concept of a Person. The journal of philosophy, 68(1), 5-20. Article

FRANKFURT, Harry G. 1988a. Identification and Wholeheartedness. In The Importance of What We Care About: Philosophical Essays. Cambridge: Cambridge University Press. 159-176. Livre FRANKFURT, Harry G. 1988b. Identification and externality. In The Importance of What We Care About: Philosophical Essays. Cambridge: Cambridge University Press. 58-68. Livre

FRANKFURT, Harry G. 1999. The Faintest Passion. Necessity, Volition, and Love. Cambridge: Cambridge University Press. 95107. Livre

GIBERT, Martin. 2009. Une pensée peut-elle être mauvaise ? Igitur. Arguments philosophiques, 1(3), 1-17.

GRECO, Daniel. 2014. A Puzzle About Epistemic Akrasia. Philosophical Studies, 167(2), 201-219. Article

HABER, Stéphane. 2007. L'aliénation. Vie sociale et expérience de la dépossession dans la théorie sociale. Paris: PUF.

HIERONYMI, Pamela. 2008. Responsibility for believing. Synthese, 161(3), 357-373. Article

HINSHELWOOD, Alec. 2013. The Relations Between Agency, Identification, and Alienation. Philosophical Explorations, 16(3),

\section{Alí́nation doxastique, CONTRÔLE ET EXPRESSIVITÉ}

243-258. Article

HOOKWAY, Christopher. 2001. Epistemic akrasia and epistemic virtue. In FAIRWEATHER Abrol et ZAGZEBSKI Linda (dir.). Virtue Epistemology: Essays on Epistemic Virtue and Responsibility. Oxford: Oxford University Press. 178-199.

HUNTER, David. 2011. Alienated Beliefs. Dialectica, 65(2), 221240. Article

KELLY, Thomas. 2002. The Rationality of Belief and Some Other Propositional Attitudes. Philosophical Studies, 110(2), 163-196. Article

KELLY, Thomas. 2014. Evidence. The Stanford Encyclopedia of Philosophy (Fall 2014 Edition), Edward N. Zalta (ed.), URL =

$<$ http://plato.stanford.edu/archives/fall2014/entries/evidence/>. LIVET, Pierre. 2002. Émotions et rationalité morale. Paris: PUF. Livre

MORAN, Richard. 2002. Frankfurt on Identification: Ambiguities of Activity in Mental Life. In BUSS Sarah (dir.). The Contours of Agency: Essays on Themes from Harry Frankfurt. Cambridge MA: Bradford Book, MIT Press. 189-217.

MORAN, Richard. 2013. Autorité et aliénation. Essai sur la connaissance de soi. Paris: Vrin.

MULLIGAN, Kevin. 1998. From Appropriate Emotions to Values. The Monist, 81(1), 161-188. Article

NAGEL, Thomas. 1974. What is it Like to Be a Bat? The Philosophical Review, 83(4), 435-450. Article

OGIEN, Ruwen. 2013. L'État nous rend-il meilleurs? Essai sur la liberté politique. Paris: Gallimard.

OWENS, David J. 2000. Reason Without Freedom. The Problem of Epistemic Normativity. London and New York: Routledge. Livre

OWENS, David J. 2002. Epistemic Akrasia. The Monist, 85(3), 381-97. Article

PAUL, Sarah. 2015. Doxastic Self-Control.American Philosophical Quarterly, 52(2), 145-58.

PETTIT, Philip, SMITH, Michael. 1996. Freedom in Belief and Desire. Journal of Philosophy, 93(9), 429-449. Article

PETTIT, Philip. 2004. Républicanisme. Une théorie de la liberté et du gouvernement. Paris: Gallimard. Livre

POLLOCK, John. 1986. Contemporary Theories of Knowledge, Towota. NJ: Rowman And Littlefield Publishers.

POUIVET, Roger. 2008. Vertus épistémiques, émotions cognitives et éducation. Éducation et didactique, 2(3). 123-139. Article RAILTON, Peter. 1994. Truth, Reason and the Regulation of Belief. Philosophical Issues, 5, 71-93. Article

REISNER, Andrew. 2009. The possibility of pragmatic reasons for belief and the wrong kind of reasons problem. Philosophical Studies, 145(2), 257-272. Article

RIBEIRO, Brian. 2011. Epistemic Akrasia. International Journal for the Study of Skepticism, 1(1), 18-25. Article

SCHWITZGEBEL, Eric. 2002. A Phenomenal, Dispositional Account of Belief. Noûs, 36(2), 249-275. Article

SCHWITZGEBEL, Eric. 2010. Acting Contrary to Our Professed Beliefs or the Gulf Between Occurrent Judgment and Dispositional Belief. Pacific Philosophical Quarterly, 91(4), 531-553. Article SEARLE, John R. 1985. L'Intentionalité. Essai de philosophie des 
états mentaux. Paris: Éditions de Minuit.

SETIYA, Kieran. 2008. Believing at Will. Midwest Studies in Philosophy, 32(1), 35-52. Article

STROUD, Sarah. 2006. Epistemic Partiality in Friendship. Ethics, 116(3), 498-524. Article

TAPPOLET, Christine. 2000. Émotions et valeurs. Paris: PUF. Livre

WATSON, Gary. 2008. L'agentivité libre. In JOUAN Marlène (dir.). Psychologie morale. Paris: Vrin. 155-178.

WEBER, Max. 1959. Le savant et le politique, Paris: Plon. Livre WILLIAMS, Bernard. 1973. Deciding to believe. Problems of the Self. Cambridge: Cambridge University Press. 136-151. Livre WILLIAMS, Bernard. 1979. Internal and External Reasons. In HARRISON Ross (dir.). Moral Luck. Cambridge: Cambridge University Press. 101-113. Livre

WILLIAMS, Bernard. 1991. L'Éthique et les limites de la philosophie. Paris: Gallimard.

Article initialement soumis le 9 mars 2016

Article révisé soumis le 31 octobre 2016.

Article accepté le 24 novembre 2016.

SITE WEB DE LA REVUE

sites.uclouvain.be/latosensu/index.php/latosensu/index

ISSN 2295-8029

DOI HTTP://DX.DOI.ORG/10.20416/LSRSPS.V4I1.513

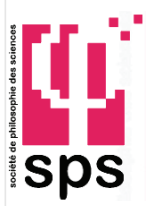

SOCIÉTÉ DE PHILOSOPHIE DES SCIENCES (SPS)

École normale supérieure

45, rue d'Ulm

75005 Paris

www.sps-philoscience.org

CONTACT ET COORDONNÉES :

Olivier Ouzilou

Université de Lorraine / Archives Henri Poincaré

Département de philosophie

91 avenue de la Libération 54001 Nancy

olivier_ouzilou@yahoo.com 